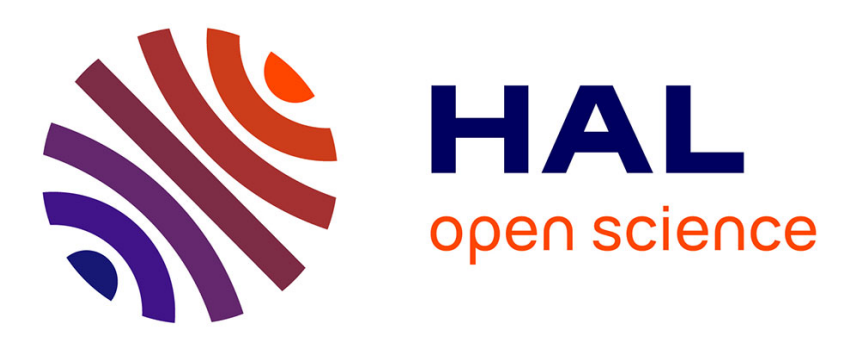

\title{
A sequential data assimilation approach for the joint reconstruction of mantle convection and surface tectonics
}

\author{
M Bocher, N Coltice, Alexandre Fournier, Paul J. Tackley
}

\section{To cite this version:}

M Bocher, N Coltice, Alexandre Fournier, Paul J. Tackley. A sequential data assimilation approach for the joint reconstruction of mantle convection and surface tectonics. Geophysical Journal International, 2015, 204 (1), pp.200-214. 10.1093/gji/ggv427 . hal-01355793

\section{HAL Id: hal-01355793 \\ https://hal.science/hal-01355793}

Submitted on 24 Aug 2016

HAL is a multi-disciplinary open access archive for the deposit and dissemination of scientific research documents, whether they are published or not. The documents may come from teaching and research institutions in France or abroad, or from public or private research centers.
L'archive ouverte pluridisciplinaire HAL, est destinée au dépôt et à la diffusion de documents scientifiques de niveau recherche, publiés ou non, émanant des établissements d'enseignement et de recherche français ou étrangers, des laboratoires publics ou privés. 


\title{
A sequential data assimilation approach for the joint reconstruction of mantle convection and surface tectonics
}

\author{
M. Bocher ${ }^{a, *}$, N. Coltice ${ }^{a, b}$, A. Fournier ${ }^{d}$, P.J. Tackley ${ }^{c}$ \\ a Laboratoire de Géologie de Lyon, Université Claude Bernard Lyon 1, \\ Ecole Normale Supṕrieure de Lyon, France. \\ ${ }^{b}$ Institut Universitaire de France, France. \\ Institute of Geophysics, E.T.H. Zurich,Switzerland. \\ ${ }^{d}$ Institut de Physique du Globe de Paris, Sorbonne Paris Cité, Université \\ Paris Diderot, CNRS (UMR 7154), Paris, France
}

Cite as Bocher, M., Coltice, N., Fournier, A., Tackley, P.J. (2016). A sequential data assimilation approach for the joint reconstruction of mantle convection and surface tectonics. Geophysical Journal International, 204(1), $200-214$.

\begin{abstract}
With the progress of mantle convection modelling over the last decade, it now becomes possible to solve for the dynamics of the interior flow and the surface tectonics to first order. We show here that tectonic data (like surface kinematics and seafloor age distribution) and mantle convection models with plate-like behaviour can in principle be combined to reconstruct mantle convection. We present a sequential data assimilation method, based on suboptimal schemes derived from the Kalman filter, where surface velocities and seafloor age maps are not used as boundary conditions for the flow, but as data to assimilate. Two stages (a forecast followed by an analysis) are repeated sequentially to take into account data observed at different times. Whenever observations are available, an analysis infers the most probable state of the mantle at this time, considering a prior guess (supplied by the forecast) and the new observations at hand, using the classical best linear unbiased estimate. Between two observation times, the evolution of the mantle is governed by the forward model of mantle convection. This method is applied to synthetic 2-D spherical annulus mantle cases to evaluate its efficiency. We compare the reference evolutions to the estimations obtained by data assimilation. Two parameters control the behaviour of the scheme: the time between two analyses, and the amplitude of noise in the synthetic observations. Our technique proves to be efficient in retrieving temperature field evolutions provided the time between two analyses is $10 \mathrm{Myr}$. If the amplitude of the a priori error on the observations is large (30 per cent), our method provides a better estimate of surface tectonics than the observations, taking advantage of the information within the physics of convection.
\end{abstract}

\section{Introduction}

Global tectonics is a surface expression of mantle convection Bercovici, 2003]: the mo-

\footnotetext{
${ }^{*}$ Corresponding author: M. Bocher Bocher)
}

tions of continents and seafloor are generated by forces acting within the mantle and the lithosphere. For example, Ricard et al. [1989] and Alisic et al. [2012] obtained a consistent description of surface kinematics by converting the long wavelength heterogeneities of seismic velocity into buoyancy forces. Mantle convection 
studies also attest to this link, showing for example that a downwelling in a context of largescale convection draws continents to aggregate [Zhong, 2001, Rolf et al., 2014].

Reconstructing the convective history of the Earth's mantle is a a long-standing problem in geosciences [Bunge et al., 1998]. Indeed, an accurate determination of mantle temperature and velocity fields evolution only for the last $200 \mathrm{My}$ would already lead to a better understanding of ore deposits formation, water resources distribution, the evolution of the geodynamo on geological time scales or deep material properties. The geodynamics community has taken advantage of the link between mantle convection and tectonics to build a first approach to reconstruct the convection history of the past $100 \mathrm{My}$. For this approach, plate tectonics theory is used to describe surface kinematics. By driving convection currents at the surface with plate kinematic reconstructions, it is possible to propose a scenario of the evolution of mantle heterogeneities. Results so obtained proved to be relatively consistent with long wavelength seismic tomography or/and the geoid [Bunge and Grand, 2000, Flament et al. 2013]. These 3D spherical convection models are called mantle circulation models [Bunge et al., 2002], as a reference to oceanography. They require very fine parametrization in order to generate internal structures consistent with both the physics of convection and geophysical observations [Bower et al., 2015]. To go one step further, several groups used tomographic models as data, in addition to plate reconstructions [Bunge et al., 2003, Ismail-Zadeh et al., 2007, Liu and Gurnis, 2008]. A temperature field of the present-day Earth's mantle is generated from these tomographic models, and the past mantle circulation is retrieved following various methods such as backward advection, variational methods and quasi-reversibility methods (see Ismail-Zadeh and Tackley [2010] for a full report on these methods). There are two drawbacks in this methodology, both difficult to overcome. First, the convection models used so far do not naturally produce Earth-like tectonics: they generate either sluggish tectonics at the surface or a stagnant lid when larger viscosity contrasts are prescribed [Solomatov, 1995]. Second, although tectonic data are the most important source of information (they are timedependent, unlike seismic data), they are used only to drive the flow, not as data to match. To drive the flow, the kinematics needs to be known at every time-step of the calculation. This is not the case for plate tectonic reconstructions, even though Gurnis et al. [2012] designed a procedure to interpolate the plate tectonics geometry between times at which it is known.

Significant progress has been made in the past 10 years on modelling convection that produces more realistic surface tectonics. Convection with a pseudo-plastic rheology generates surface tectonics with a plate-like behaviour [Moresi and Solomatov, 1998, Moresi et al., 2000, Stein et al., 2004, Bercovici, 2003, Tackley, 2000, Van Heck and Tackley, 2008, Bercovici and Ricard, 2014]. Recent models display seafloor spreading and continental drift comparable to that of the Earth to first order: seafloor age distributions and the time scale of spreading fluctuations are consistent with what has been inferred for the Earth for the last 200 My Coltice et al., 2012, 2013]. This opens the way to producing a mantle circulation model using convection models with plate-like behaviour, under the observational constraint provided by tectonic data. This constraint is not to be enforced through kinematic boundary conditions imposed at every time step. Instead, it should be taken into account in a statistically consistent fashion, whereby the prediction due to the model and the observations are combined in a way that respects the uncertainties affecting both.

We explore here this possibility using a controlled case, and present a proof of concept. We have developed a sequential data assimilation method which is a suboptimal scheme based on the Kalman filter (see Todling and Cohn [1994] for a review of suboptimal schemes based on the Kalman filter). Such data assimilation methodology provides the best linear unbiased estimate of the temperature field and surface velocities at times data are available, and a prediction of the evolution of the system state between those times. Using synthetic tests in spherical annulus geometry [Hernlund and Tackley, 2008], we show that our data assimilation method is efficient for reconstructions over 200 My with data gaps of $5-10 \mathrm{My}$ and relative uncertainties on 
surface observations below $30 \%$. This method also allows the refinement of surface tectonics, taking advantage of the information within the physics of convection.

In the following, we first describe the general approach to Kalman Filtering in Section 2. Section 3 provides details on the definition of the different operators and vectors forming the backbone of a sequential data assimilation framework: the physical model, the data and the state for mantle convection, as well as the observation operator. Section 4 presents our sequential data assimilation algorithm and the adjustments used for the reconstruction of mantle convection. Then, we present in Section 5 an evaluation of the method with a series of synthetic experiments, which leads to the discussion of section 6 .

\section{The Extended Kalman Filter}

We introduce in this section the Extended Kalman Filter that we adapt and apply to the convection reconstruction problem. We use here classical notations, taken from Ide et al. [1997]. Our goal is to estimate the evolution of the mantle temperature field, using information from a mantle convection model and tectonic data including global kinematics and seafloor ages. The following is a rather general description of the Extended Kalman Filter; similar developments can be found in Ghil and MalanotteRizzoli [1991], Kalnay [2003], Wunsch [2006] for meteorology and oceanography and in Fournier et al. 2010 for geomagnetism, for example.

Data assimilation aims at estimating the state of a dynamical system and its evolution for a given period of time, combining information from observations and a physical model. The evolution of the state of the system is described by the set of true state vectors, storing the chosen scalar fields that are discretized:

$$
\left\{\boldsymbol{x}_{1}^{t}, \boldsymbol{x}_{2}^{t}, \ldots, \boldsymbol{x}_{n_{t}}^{t}\right\},
$$

the subscripts $\left\{1,2, \ldots, n_{t}\right\}$ being the discrete time steps.

Likewise, the observations are defined as a set of column vectors

$$
\left\{\boldsymbol{y}_{t_{1}}^{o}, \boldsymbol{y}_{t_{2}}^{o}, \ldots, \boldsymbol{y}_{t_{n}}^{o}\right\} \text { with }\left\{t_{1}, t_{2}, \ldots, t_{n}\right\} \subset\left\{1,2, \ldots, n_{t}\right\}
$$

The true state and the observations are linked linearly by the observation operator $\boldsymbol{H}$

$$
\forall t_{i} \in\left\{t_{1}, t_{2}, \ldots, t_{n}\right\}, \quad \boldsymbol{y}_{t_{i}}^{o}=\boldsymbol{H} \boldsymbol{x}_{t_{i}}^{t}+\boldsymbol{\epsilon}_{t_{i}}^{o},
$$

where $\epsilon_{t_{i}}^{o}$ is the observation error at the timestep $t_{i}$. The precise value of $\boldsymbol{\epsilon}_{t_{i}}^{o}$ is unknown. However, it is possible to model it as a random vector and estimate its probability density function (PDF). We assume $\epsilon_{t_{i}}^{o}$ follows a centred Gaussian statistics, and its associated covariance matrix

$$
\boldsymbol{R}_{t_{i}}=\operatorname{Covar}\left(\epsilon_{t_{i}}^{o}\right)=\left\langle\epsilon_{t_{i}}^{o}\left(\epsilon_{t_{i}}^{o}\right)^{T}\right\rangle
$$

is called the observation error covariance matrix. The operator []$^{T}$ means transpose. The Extended Kalman Filter belongs to the Sequential Data Assimilation techniques. It consists of integrating the observation vectors one after the other into a numerical model, when they become available. Fig. 1 gives an overview of this procedure. The initialization, at timestep $t_{1}$, provides an a priori guess of the state of the system. Then two steps called analysis and forecast are performed sequentially, every time a new observation vector becomes available, until all the observations have been taken into account. Hence the name of the technique: sequential data assimilation. The analysis corrects the current estimate of the system state by considering the new observations. The forecast provides an estimation of the evolution of the system until the next time new observations are available. The evolution of the estimated state should converge towards the true state as more observations are assimilated.

\subsection{Initialization}

The Extended Kalman Filter algorithm starts at the timestep $t_{1}$, when the first observations are available. Before taking into account the first observations, an a priori state of the system has to be estimated. It is called the background state $\boldsymbol{x}^{b}$. The relationship between the background state and the true state at $t_{1}$ is

$$
\boldsymbol{x}_{b}=\boldsymbol{x}_{t_{1}}^{t}+\boldsymbol{\epsilon}^{b},
$$

where $\epsilon^{b}$ is the background state error. $\epsilon^{b}$ is a random vector with $\left\langle\boldsymbol{\epsilon}^{b}\right\rangle=\mathbf{0}$ and $\operatorname{Covar}\left(\boldsymbol{\epsilon}^{b}\right)=$ $\boldsymbol{P}^{b}$. 


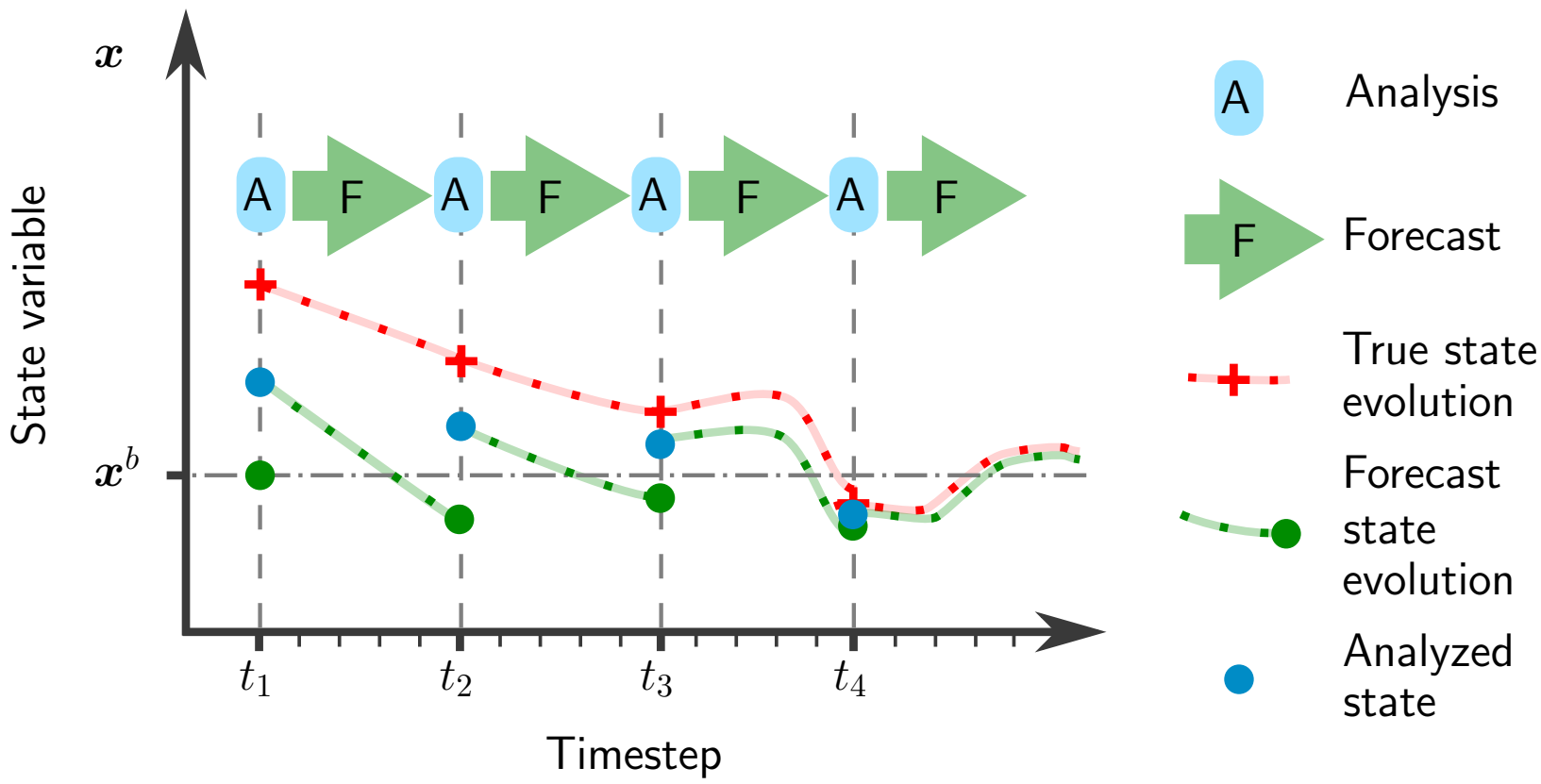

Figure 1: Principle of Sequential Data Assimilation. The initialization at time $t_{1}$ sets the background state $\boldsymbol{x}^{b}$ (green dot at $\left.t_{1}\right) . \boldsymbol{x}^{b}$ is corrected by taking into account new observations, leading to the first analyzed state $\boldsymbol{x}_{t_{1}}^{a}$ (blue dot at $t_{1}$ ), which is closer to the true state $\boldsymbol{x}_{t_{1}}^{t}$ (red cross at $t_{1}$ ). The evolution of the state (green line) is then computed by the model $\mathcal{M}$ until $t_{2}$, leading to a new forecast state $\boldsymbol{x}_{t_{2}}^{f}$ (green dot). A new analyzed state $\boldsymbol{x}_{t_{2}}^{a}$ is computed considering new observations. The sequence is repeated until all observations have been taken into account. The aim of data assimilation is to get as close as possible to the true state evolution (red line).

The background state vector is considered as the forecast state vector at timestep $t_{1}$, written $\boldsymbol{x}_{t_{1}}^{f}$ and its covariance matrix $\boldsymbol{P}^{b}$ is the forecast error covariance matrix at $t_{1}$, written $\boldsymbol{P}_{t_{1}}^{f}$.

\subsection{Analysis and forecast sequence}

At $t_{i}$, a new estimate of the state of the system is calculated considering $\boldsymbol{y}_{t_{i}}^{o}, \boldsymbol{R}_{t_{i}}, \boldsymbol{x}_{t_{i}}^{f}$ and $\boldsymbol{P}_{t_{i}}^{f}$. This step is called analysis, the new estimate being the analyzed state $\boldsymbol{x}_{t_{i}}^{a}$. The relationship between the analyzed state and the true state is

$$
\boldsymbol{x}_{t_{i}}^{a}=\boldsymbol{x}_{t_{i}}^{t}+\boldsymbol{\epsilon}_{t_{i}}^{a},
$$

where $\epsilon_{t_{i}}^{a}$ is the analysis error at $t_{i} . \epsilon_{t_{i}}^{a}$ is modelled as a random vector following a Gaussian law, and its covariance $\boldsymbol{P}_{t_{i}}^{a}$ is the analysis error covariance matrix. The analyzed state $\boldsymbol{x}_{t_{i}}^{a}$ is chosen so that

$\boldsymbol{x}_{t_{i}}^{a}$ is a linear combination of $\boldsymbol{x}_{t_{i}}^{f}$ and $\boldsymbol{y}_{t_{i}}^{o}$,

$\operatorname{Tr}\left(\boldsymbol{P}_{n}^{a}\right)$ is minimised,

$$
\left\langle\boldsymbol{\epsilon}_{n}^{a}\right\rangle=\mathbf{0}
$$

These conditions lead to the classical Best Linear Unbiased Estimate (see Ghil and Malanotte-Rizzoli [1991] for example)

$$
\begin{aligned}
\boldsymbol{x}_{t_{i}}^{a} & =\boldsymbol{x}_{t_{i}}^{f}+\boldsymbol{K}_{t_{i}}\left[\boldsymbol{y}_{t_{i}}^{o}-\boldsymbol{H} \boldsymbol{x}_{t_{i}}^{f}\right], \\
\boldsymbol{K}_{t_{i}} & =\left(\boldsymbol{H} \boldsymbol{P}_{t_{i}}^{f}\right)^{T}\left[\boldsymbol{H}\left(\boldsymbol{H} \boldsymbol{P}_{t_{i}}^{f}\right)^{T}+\boldsymbol{R}\right]^{-1}, \\
\boldsymbol{P}_{t_{i}}^{a} & =\left(\boldsymbol{I}-\boldsymbol{K}_{t_{i}} \boldsymbol{H}\right) \boldsymbol{P}_{t_{i}}^{f},
\end{aligned}
$$

where $\boldsymbol{K}_{t_{i}}$ is the Kalman gain at $t_{i}$. If the probability density functions of the errors on the forecast state and the observed data are Gaussian and the observation operator is linear, then $\boldsymbol{x}_{t_{i}}^{a}$ is not only the estimate of minimum variance but as well the most likely state: in this case, the analysis will be optimal.

The evolution of the estimated state of the system from $t_{i}$ to $t_{i+1}$, the next timestep observations are available, is computed using the direct numerical model $\mathcal{M}$ :

$$
\boldsymbol{x}_{t_{i+1}}^{f}=\mathcal{M}\left(\boldsymbol{x}_{t_{i}}^{a}\right)+\boldsymbol{\eta}_{t_{i}} .
$$

Its associated covariance matrix is

$$
\boldsymbol{P}_{t_{i+1}}^{f}=\boldsymbol{M} \boldsymbol{P}_{t_{i}}^{a} \boldsymbol{M}^{T}+\boldsymbol{Q}_{t_{i}}
$$


where $M$ is the tangent linear model, i.e. the linearised version of the operator $\mathcal{M}$ and $Q_{t_{i}}$ is the model error covariance matrix. Equations 10 and 11 describe the forecast step. The estimated state of the system at a timestep $n \boldsymbol{x}_{n}^{f}$ is called the forecast state and $\boldsymbol{P}_{t_{i+1}}^{f}$ is the forecast error covariance matrix at timestep $t_{i+1} . \boldsymbol{P}_{t_{i+1}}^{f}$ takes into account both the propagation of errors of the analyzed state at timestep $t_{i}, \boldsymbol{\epsilon}_{t_{i}}^{a}$ and the model errors $\boldsymbol{\eta}_{t_{i}}$. We assume a perfect model in this study, so that

$$
\forall t_{i}, \quad \boldsymbol{\eta}_{t_{i}}=\mathbf{0} .
$$

\section{Convection model, Mantle State Vector and Tectonic Data}

In this section, we describe in detail what $\mathcal{M}$, $\boldsymbol{H}, \boldsymbol{x}$ and $\boldsymbol{y}$ refer to in our mantle convection context.

\subsection{Convection Model with Plate-Like Be- haviour}

The forward model $\mathcal{M}$ is our source of prior information. It solves the equations of conservation of mass, momentum and energy with classical simplifications for mantle convection: infinite Prandtl number and Boussinesq approximation. We further assume an isochemical mantle, and non-dimensionalize the equations to thermal diffusion scales (for a full development of the equations, see Ricard [2007] for example). We obtain

$$
\begin{aligned}
& \nabla \cdot \boldsymbol{u}=0, \\
& \nabla \cdot\left[\mu\left(\nabla \boldsymbol{u}+(\nabla \boldsymbol{u})^{T}\right)\right]-\nabla p+\operatorname{Ra} T \boldsymbol{e}_{r}=0, \\
& \frac{D T}{D t}=\nabla^{2} T+H,
\end{aligned}
$$

where $\boldsymbol{u}, p, T$ and $t$ are the non-dimensional velocity, dynamic pressure, temperature and time, respectively. We work in spherical coordinates $(r, \theta, \phi)$ of unit vectors $\left(\boldsymbol{e}_{r}, \boldsymbol{e}_{\theta}, \boldsymbol{e}_{\phi}\right)$. Ra is the Rayleigh number and $H$ is the non-dimensional internal heating rate. The models presented here have $10 \%$ basal heating and $90 \%$ internal heating.

The temperatures at the top and bottom boundaries are set to $T_{a}$ and $T_{b}$. The surface and the base of the model are shear-stress free.
The dynamic viscosity $\mu$ varies with temperature and stress following the equation

$$
\mu=\left(\mu_{T}^{-1}+\mu_{y}^{-1}\right)^{-1},
$$

$\mu_{T}$ decreasing exponentially with temperature (according to Arrhenius law), and divided by $\beta$ when reaching the solidus:

$$
\begin{aligned}
& \mu_{T}=\exp \left(\frac{E_{A}}{T+T_{1}}-\frac{E_{A}}{2 T_{1}}\right) \quad \text { if } \quad T<T_{s}, \\
& \mu_{T}=\beta^{-1} \exp \left(\frac{E_{A}}{T+T_{1}}-\frac{E_{A}}{2 T_{1}}\right) \quad \text { if } \quad T>T_{s} .
\end{aligned}
$$

$T_{1}$ is the temperature for which $\mu_{T}=1, E_{A}$ is the activation energy and $T_{s}=T_{s_{0}}+\nabla_{r} T_{s}\left(r_{a}-\right.$ $r$ ) with $r_{a}$ the surface value of $r$. $T_{s}$ models the variation of solidus with depth and is tuned so that the viscosity drop is located at the base of the top boundary layer. This results in a weaker asthenosphere and favours plate-like behaviour [Richards et al., 2001, Tackley, 2000].

$\mu_{y}$ is defined by

$$
\sigma_{y i e l d}=\sigma_{Y}+\left(r_{a}-r\right) \cdot \nabla_{r} \sigma_{Y}, \quad \mu_{y}=\frac{\sigma_{y i e l d}}{2 \dot{\epsilon}},
$$

with $\sigma_{Y}, \nabla_{r} \sigma_{Y}$ and $\dot{\epsilon}$ being the yield stress at the surface, the depth-dependence of the yield stress and the second invariant of the strain rate tensor respectively. The strain rate tensor is linked to the velocity by

$$
\dot{\boldsymbol{\epsilon}}=\frac{1}{2}\left[\boldsymbol{\nabla} \boldsymbol{u}+(\boldsymbol{\nabla} \boldsymbol{u})^{T}\right] .
$$

Solutions are computed using StagYY [Tackley et al., 1993], a finite-volume, multigrid convection code. We use a spherical annulus grid which provides results closer to the spherical grid than a cylindrical geometry Hernlund and Tackley, 2008]. The grid is refined in the radial direction near the upper boundary of the model. In the following, the longitudinal coordinate of a point is written $\phi_{m}$, with $m \in\{1,2, \ldots, M\}$ and its radial coordinate is written $r_{n}$ with $n \in$ $\{1,2, \ldots, N\}, r$ varying from $r_{b}$ to $r_{a}$. The value of the parameters used for this work are given in Table 1

Since this work is a proof of concept, we chose a fairly simple model, with equation governing the flow relying on strong assumptions (incompressible, isochemical mantle). The Rayleigh 
Table 1: Values of the parameters of the forward models

\begin{tabular}{|c|l|c|c|c|}
\hline Symbol & Meaning & model 1 & model 2 & model 3 \\
\hline$R a$ & Rayleigh number & $10^{6}$ & $10^{6}$ & $10^{7}$ \\
\hline$H$ & Non-dimensional internal heating rate & 20.5 & 13.5 & 20.5 \\
\hline$M$ & Resolution of the grid in the longitude direction & 384 & 384 & 512 \\
\hline$N$ & Resolution of the grid in the radial direction & 48 & 48 & 64 \\
\hline$r_{a}$ & Radius of the top of the domain & 2.2 & 2.2 & 2.2 \\
\hline$r_{b}$ & Radius of the bottom of the domain & 1.2 & 1.2 & 1.2 \\
\hline$T_{a}$ & Temperature at the top of the domain & 0 & 0 & 0 \\
\hline$T_{b}$ & Temperature at the bottom of the domain & 0.9 & 1.3 & 1 \\
\hline$E_{A}$ & Activation Energy & 23.03 & 23.03 & 23.03 \\
\hline$T_{1}$ & Temperature at which $\mu_{T}=1$ & 1 & 1 & 1 \\
\hline$\beta$ & Factor of viscosity reduction for partial melting & 10 & 10 & 10 \\
\hline$T_{s_{0}}$ & Solidus Temperature at $r=r_{a}$ & 0.6 & 0.6 & 0.6 \\
\hline$\nabla_{r} T_{s}$ & Radial gradient of the solidus temperature & 2 & 2 & 2 \\
\hline$\sigma_{Y}$ & Yield Stress & $1.10^{4}$ & $1.10^{4}$ & $4.10^{4}$ \\
\hline$\nabla_{r} \sigma_{Y}$ & Radial gradient of the yield stress & 0.025 & 0.025 & 0.025 \\
\hline
\end{tabular}

number is $10^{6}$ which is one or two orders of magnitude lower than that of the Earth, but high enough to ensure chaotic convection. The viscosity law self-consistently generates plates at the surface, as it can be seen from the surface velocity in Fig 10 . Ignoring many more complexities does not mean they are not fundamental for reconstructing Earth mantle evolution, but we focus in this manuscript on the data assimilation methodology.

\subsection{The State of the mantle}

Given Equations 13 to 15 above, the field variables describing the state of the Earth's mantle $\boldsymbol{x}$ are velocity, temperature and pressure. Inertial forces are negligible for mantle convection, which means that the velocity and pressure fields can be deduced from the temperature field at any time given the viscosity law and other parameters, as shown in the diagnostic Equations 13 and 14 . Since it is possible to compute $\boldsymbol{u}$ and $p$ from the sole knowledge of $T, \boldsymbol{x}$ should be restricted to $T$ alone.

\subsection{The Data: Surface Heat Flux and Surface velocities}

As a first approach, the data $\boldsymbol{y}^{o}$ we use are not direct measurements per se, but plate reconstruction models. For instance, Seton et al. 2012 or Shephard et al. 2013 proposed plate tectonics reconstructions for the last $200 \mathrm{My}$, using the continuously closing plates methodology Gurnis et al. 2012] so that a reconstruction can be numerically computed for any time between a given $t_{i}$ and the subsequent $t_{i+1}$. These reconstructions integrate paleomagnetic, paleobiological and geological data to provide continuous maps of surface velocity and seafloor age as well as the position and geometry of continents. It is this type of data that is used today in convection reconstructions with imposed boundary conditions. One fundamental difference between these methods and our sequential assimilation method is that the latter naturally takes into account uncertainties in the reconstructions. A second difference is that we do not need the surface data to be known at all times.

Plate reconstructions provide estimates of the velocity at any location on the surface of the Earth in the approximation of the plate tectonics theory, as well as the age of the seafloor. In the model we use in this manuscript, surface heat flux is an excellent proxy for the age of the seafloor Coltice et al. 2012. Consequently, we propose to consider surface heat flux and surface velocity as the data to assimilate. However, with more sophisticated models, small scale convection would require an explicit computation of the age of the seafloor.

At each time $t_{i}$, the data vector for the present 
study will be

$$
\boldsymbol{y}=\left[q_{s}\left(\phi_{1}\right), \ldots, q_{s}\left(\phi_{N}\right), V_{s}\left(\phi_{1}\right), \ldots, V_{s}\left(\phi_{N}\right)\right]^{T},
$$

where $q_{s}\left(\phi_{m}\right)$ and $V_{s}\left(\phi_{m}\right)$ are the surface heat flux and tangential velocity values at longitude $\phi_{m}$, respectively. Hence, for the model parameters set of Table 1, the data vector contains $2 N=768$ values.

\subsection{The Observation Operator and the Aug- mented State}

As described by Equation 3, the link between the state of the system $\boldsymbol{x}$ and the data $\boldsymbol{y}$ has to be expressed in the form of a linear equation. This is straightforward for surface heat flux. At a given longitude $\phi_{m}$, the surface heat flux is approximated by a first order discretization of Fourier's law

$$
\forall \phi_{m} \quad q\left(\phi_{m}\right)=-k \frac{T\left(\phi_{m}, r_{N}\right)-T\left(\phi_{m}, r_{N-1}\right)}{r_{N}-r_{N-1}},
$$

where $k$ is the thermal conductivity, which is 1 for our non-dimensional framework.

However, the link between the velocity field and the temperature field is highly nonlinear, because of our choice of rheology. This is why we consider an augmented state of the mantle, containing both temperature and surface velocity fields:

$$
\begin{aligned}
\boldsymbol{x}= & {\left[T\left(\phi_{1}, r_{1}\right), \ldots, T\left(\phi_{M}, r_{1}\right), T\left(\phi_{1}, r_{2}\right), \ldots,\right.} \\
& \left.T\left(\phi_{M}, r_{N}\right), V\left(\phi_{1}\right), \ldots, V\left(\phi_{M}\right)\right],
\end{aligned}
$$

where $T\left(\phi_{m}, r_{n}\right)$ is the temperature value at longitude $\phi_{m}$ and radius $r_{n}$ and $V\left(\phi_{m}\right)$ is the surface tangential velocity value at longitude $\phi_{m}$. For the model parameters set of Table 1, the state vector contains $N M+N=18816$ values.

\section{Sequential Data Assimilation Algorithm for Mantle Convection}

We describe here the steps of our sequential algorithm, which are

an initialization, which evaluates the background state $\boldsymbol{x}^{b}$ and its error covariance matrix $\boldsymbol{P}^{b}$, an analysis which estimates

$$
\left\{\boldsymbol{x}_{t_{i}}^{f}, \boldsymbol{P}_{t_{i}}^{f}, \boldsymbol{y}_{t_{i}}^{o}, \boldsymbol{R}_{t_{i}}^{o}\right\} \rightarrow\left\{\boldsymbol{x}_{t_{i}}^{a}\right\},
$$

a forecast, which computes

$$
\left\{\boldsymbol{x}_{t_{i}}^{a}\right\} \rightarrow\left\{\boldsymbol{x}_{t_{i+1}}^{f}, \boldsymbol{P}_{t_{i+1}}^{f}\right\} .
$$

\subsection{Initialization}

The background state $\boldsymbol{x}^{b}$ and its associated covariance matrix $\boldsymbol{P}^{b}$ describe an estimate of the state of the system when no observation is yet available. $\boldsymbol{x}^{b}$ components correspond to the average temperature field and surface velocities of the mantle. $\boldsymbol{P}^{b}$ contains the two-point spatial correlation of the temperature field and surface velocity field, as described by Balachandar [1998.

To estimate $\boldsymbol{x}^{b}$ and $\boldsymbol{P}^{b}$, we use a set of $K=200$ states of the system $\boldsymbol{x}_{k}$ with $k \in$ $\{1, \ldots, K\}$, computed with the mantle convection code. These states are extracted from a free run of the convection model (i.e. unconstrained by data). To ensure decorrelation between the states, the time between two snapshots is equivalent to 5 Lyapunov times. The Lyapunov time is the e-folding time for the growth of an initial error in a dynamic system. In the calculations we describe below, we obtain a typical Lyapunov time of $140 \mathrm{My}$, similar to the Lyapunov times computed in 3D spherical convection models [Bello et al., 2014].

The background state is

$$
\boldsymbol{x}^{b}=\langle\boldsymbol{x}\rangle=\frac{1}{K} \sum_{k=1}^{K} \boldsymbol{x}_{k}
$$

and the error covariance matrix is

$$
\boldsymbol{P}^{b}=\operatorname{Covar}(\boldsymbol{x})=\frac{1}{K-1} \sum_{k=1}^{K}\left(\boldsymbol{x}_{k}-\boldsymbol{x}^{b}\right)\left(\boldsymbol{x}_{k}-\boldsymbol{x}^{b}\right)^{T} .
$$

The probability density functions of all the variables are also estimated, to compare them to Gaussian probability density functions. Indeed, the best linear unbiased estimate used during analysis is optimal only in the linear Gaussian case, as described in section 2 . 
As noted before, the initial setting of the model is spherically symmetric. As a consequence

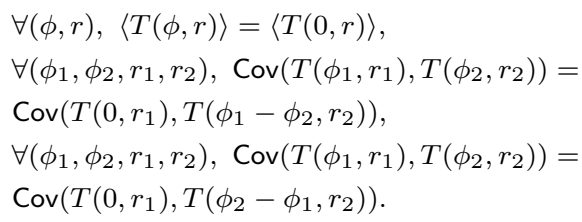

Likewise

$$
\begin{aligned}
& \forall \phi,\left\langle V_{s}(\phi)\right\rangle=\left\langle V_{s}(0)\right\rangle, \\
& \forall\left(\phi_{1}, \phi_{2}\right), \operatorname{Cov}\left(V_{s}\left(\phi_{1}\right), V_{s}\left(\phi_{2}\right)\right)= \\
& \operatorname{Cov}\left(V_{s}(0), V_{s}\left(\phi_{1}-\phi_{2}\right)\right), \\
& \forall\left(\phi_{1}, \phi_{2}, r_{1}\right), \\
& \operatorname{Cov}\left(T\left(\phi_{1}, r_{1}\right), V_{s}\left(\phi_{2}\right)\right)= \\
& \operatorname{Cov}\left(T\left(0, r_{1}\right), V_{s}\left(\phi_{2}-\phi_{1}\right)\right), \\
& \operatorname{Cov}\left(T\left(\phi_{1}, r_{1}\right), V_{s}\left(\phi_{2}\right)\right)= \\
& -\operatorname{Cov}\left(T\left(0, r_{1}\right), V_{s}\left(\phi_{1}-\phi_{2}\right)\right) .
\end{aligned}
$$

These symmetries are enforced during the computation of $\boldsymbol{x}^{b}$ and $\boldsymbol{P}^{b}$, which decreases the number $K$ of states of the system needed to obtain converged statistics. For our test case, the covariance matrix $\boldsymbol{P}^{b}$ contains $(N M+$ $N)^{2}=18816^{2}=354,041,856$ components. The symmetries in the covariances dramatically reduce the number of independant components to $N / 2(M+1)^{2}=3,557,400$.

From $\boldsymbol{x}^{b}$ and $\boldsymbol{P}^{b}$, a background data vector $\boldsymbol{y}^{b}$ and its associated covariance matrix $\boldsymbol{R}^{b}$ are defined as

$$
\begin{aligned}
\boldsymbol{y}^{b} & =\boldsymbol{H} \boldsymbol{x}^{b}, \\
\boldsymbol{R}^{b} & =\boldsymbol{H} \boldsymbol{P}^{b} \boldsymbol{H}^{T} .
\end{aligned}
$$

Figs 2 and 3 represent the PDF of surface velocity, surface heat flux and temperature for different depths for our test model described in Section 3.1. Surface velocities (Fig. 2 left) have a Gaussian PDF which is consistent with the approximation we made. On the contrary, the PDFs of temperature (Fig. 3) are more complex. For temperature values close to the surface, the PDFs are highly skewed and have a strong kurtosis. This behaviour is due to the strongly nonlinear rheology at the surface of the model (kurtosis), and the isothermal boundary conditions (skewness). As a consequence, the estimation performed during analysis will not be optimal. $\boldsymbol{x}^{b}, \boldsymbol{P}^{b}, \boldsymbol{y}^{b}$ and $\boldsymbol{R}^{b}$ are used to standardize the state $\boldsymbol{x}$ and data $\boldsymbol{y}$ for the analysis step. We define their corresponding standard score state $\widetilde{\boldsymbol{x}}$ and standard score data $\widetilde{\boldsymbol{y}}$ by

$$
\begin{aligned}
\widetilde{\boldsymbol{x}} & =\left(\boldsymbol{S}^{b}\right)^{-1}\left(\boldsymbol{x}-\boldsymbol{x}^{b}\right), \\
\widetilde{\boldsymbol{y}} & =\left(\boldsymbol{S}_{y}^{b}\right)^{-1}\left(\boldsymbol{y}-\boldsymbol{y}^{b}\right),
\end{aligned}
$$

where $\boldsymbol{S}^{b}$ is the diagonal matrix containing the standard deviations of each component of the background state and $\boldsymbol{S}_{y}^{b}$ the diagonal matrix containing the standard deviations of each component of the background data,

$$
\begin{aligned}
\boldsymbol{S}^{b} & =\operatorname{Diag}\left(\boldsymbol{P}^{b}\right)^{1 / 2}, \\
\boldsymbol{S}_{y}^{b} & =\operatorname{Diag}\left(\boldsymbol{R}^{b}\right)^{1 / 2} .
\end{aligned}
$$

The standard score observation operator $\widetilde{\boldsymbol{H}}$ is then

$$
\widetilde{\boldsymbol{H}}=\left(\boldsymbol{S}_{y}^{b}\right)^{-1} \boldsymbol{H} \boldsymbol{S}^{b} .
$$

The standardized background state $\widetilde{\boldsymbol{x}}^{b}$ and its associated covariance matrix $\widetilde{\boldsymbol{P}}^{b}$ are then

$$
\begin{aligned}
\widetilde{\boldsymbol{x}}^{b} & =\mathbf{0}, \\
\widetilde{\boldsymbol{P}}^{b} & =\left(\boldsymbol{S}^{b}\right)^{-1} \boldsymbol{P}^{b}\left(\boldsymbol{S}^{b}\right)^{-1} .
\end{aligned}
$$

The covariance matrix $\widetilde{\boldsymbol{P}}^{b}$, associated with the standardized background state corresponds as well to the correlation matrix of the background state. Equations 28 to 33 are also valid for correlations values, i.e. for the components of $\widetilde{\boldsymbol{P}}^{b}$.

Equations 28 and 29 show that the correlation between any couple of temperature variables can be summed up by plotting only the correlations

$$
\begin{aligned}
& \forall r_{1}, r_{2} \in\left[r_{b}, r_{a}\right]^{2}, \forall \Delta \phi \in[0, \pi], \\
& \operatorname{Cor}\left(T\left(0, r_{1}\right), T\left(\Delta \phi, r_{2}\right)\right)
\end{aligned}
$$

Hence, for a given $r_{1}$, the two points spatial temperature-temperature correlations can be mapped onto a half-spherical annulus. Fig. 4 represents these correlation maps for different values of $r_{1}: r_{1}=r_{b}$ (Fig. 4(a)), $r_{1}=\left(r_{a}+\right.$ $\left.r_{b}\right) / 2$ (Fig. 4(b)) and $r_{1}=r_{a}$ (Fig. 4(c)). Fig. 4(a) shows that bottom temperatures have strong correlations with neighbouring temperatures, up to 0.6 at a distance corresponding to a third of the domain depth. A fourth order 


\section{- Empirical PDF - Approximation by a Gaussian PDF}
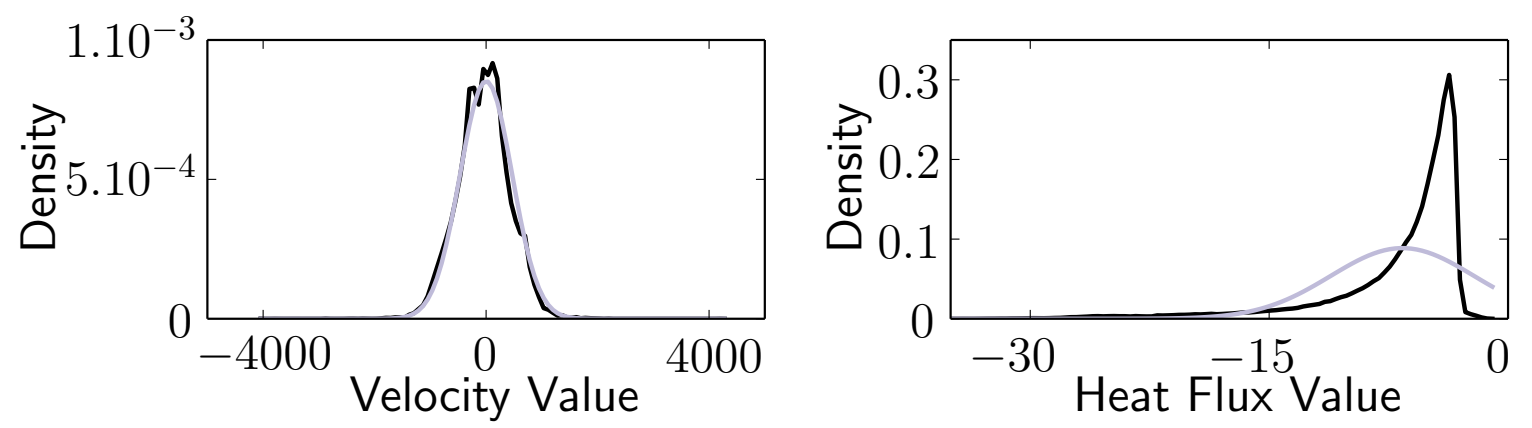

Figure 2: Probability density functions of background surface velocity (left) and background surface heat flux (right) compared to their Gaussian approximations (in light gray)
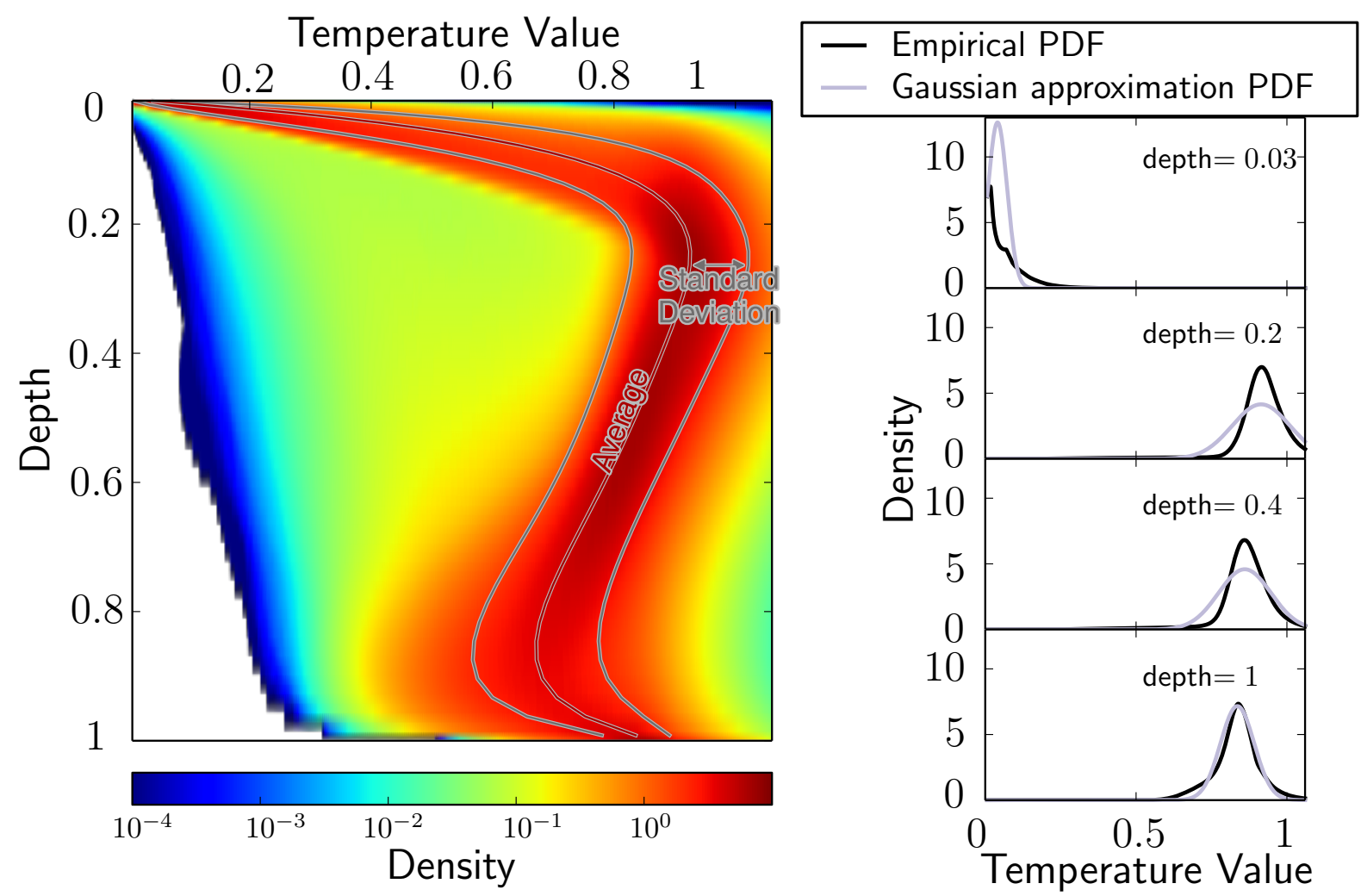

Figure 3: Map of the probability density functions of background temperature calculated at each depth (left). On the right, probability density functions for different depth are shown, so as to compare them with their Gaussian approximations used for data assimilation. 

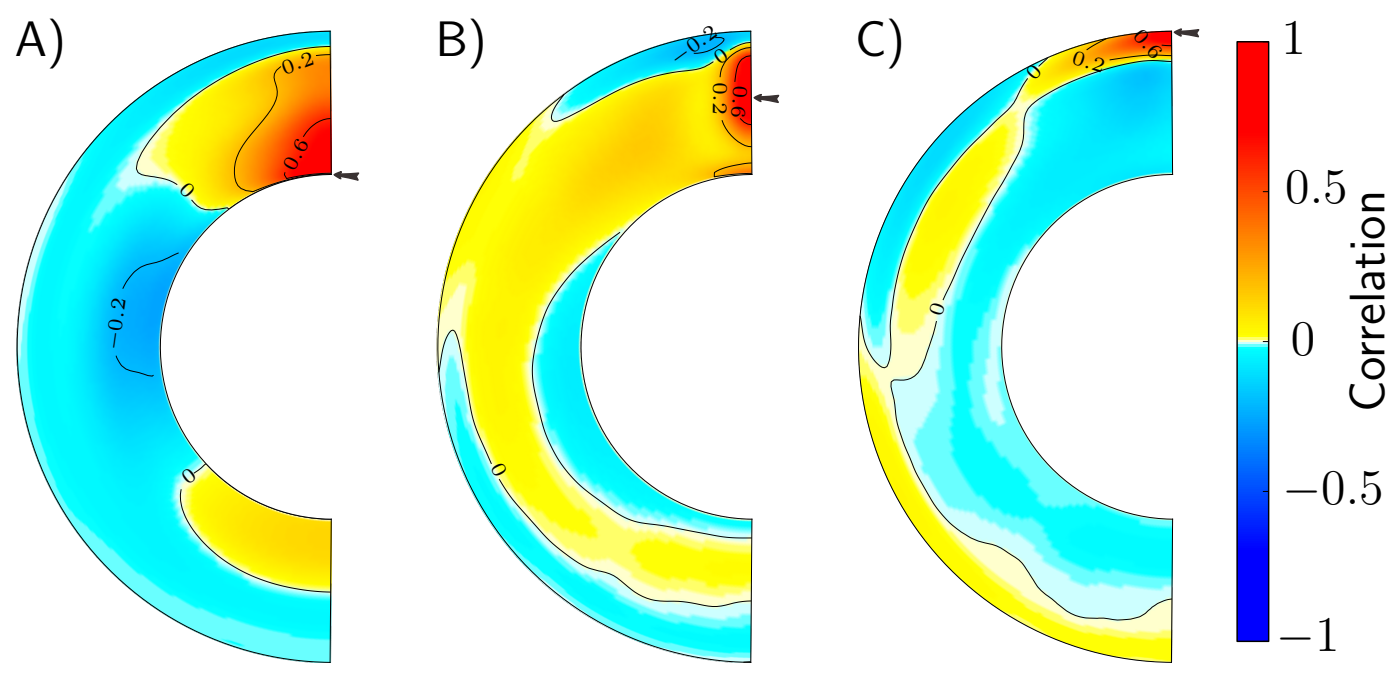

Figure 4: Maps of the two-point spatial correlation of temperature. The arrow indicates the location of the anchor point. The maps represents the correlation between the temperature at anchor points and the temperature points on the rest of the domain. Considering the symmetries of correlations described on equations 28 and 29 , only the correlations $\forall \phi^{\prime} \in[0, \pi], r^{\prime} \in[0,1], \operatorname{Cov}\left(T(0, r), T\left(\phi^{\prime}, r^{\prime}\right)\right)$ for $r=r_{b}(\mathrm{~A}), r=\left(r_{a}+r_{b}\right) / 2(\mathrm{~B})$ and $r=r_{a}(\mathrm{C})$ are shown.

convection pattern is also notable, with bottom temperature values anticorrelated with temperatures at a longitude of $+90^{\circ}$ and correlated with values at a longitude of $+180^{\circ}$. The mid-mantle temperature (Fig. 4b) has an ellipsoidal zone of high correlation, which is the result of the dominant vertical motion in this part of the domain. Finally the top temperatures have a zone of correlation which extends towards the longitudinal direction, corresponding to the dominant horizontal motion at the surface of the domain. The anticorrelation between the top boundary layer and the rest of the domain is also remarkable.

Equation 32 shows that the correlation between any couple of temperature-surface velocity variables can be summed up by plotting only the correlations

$$
\forall r \in\left[r_{b}, r_{a}\right], \forall \Delta \phi \in[0,2 \pi], \operatorname{Cor}\left(V_{s}(0), T(\Delta \phi, r)\right)
$$

Hence, the two points spatial temperaturevelocity correlations can be mapped onto a spherical annulus. Fig. 5 represents this correlation map. Fig. 5 shows that surface velocities are weakly correlated with temperature, with a maximum correlation value of 0.4 . Nevertheless, the

Figure 5: Map of the two-point spatial correlations of temperature and velocity. The arrow indicate the anchor point. The map represents the correlation between the surface velocity value at the anchor point and the temperature values on the rest of the domain. Considering the symmetries of correlations described on equations 32 only the correlations $\forall r \in\left[r_{b}, r_{a}\right], \forall \Delta \phi \in$ $[0,2 \pi], \operatorname{Cor}\left(V_{s}(0), T(\Delta \phi, r)\right)$ are shown. 
correlations between surface velocity and temperature at the bottom are not negligible, and this is mainly due to the sinking of slabs to the bottom of the model.

Although we ignore the non-Gaussianity of some distributions, second order statistics succeed in summing up the main features of the convection model dynamics.

Furthermore, the matrix $\widetilde{\boldsymbol{P}}^{b}$ is eigendecomposed leading to

$$
\widetilde{\boldsymbol{P}}^{b}=\boldsymbol{V} \boldsymbol{\Lambda} \boldsymbol{V}^{T} .
$$

where $\boldsymbol{V}$ contains the eigenvectors of $\widetilde{\boldsymbol{P}}^{b}$ and $\boldsymbol{\Lambda}$ its eigenvalues. The background correlation matrix $\widetilde{\boldsymbol{P}}^{b}$ is reduced to

$$
\widetilde{\boldsymbol{P}}_{r}^{b}=\boldsymbol{V}_{r} \boldsymbol{\Lambda}_{r} \boldsymbol{V}_{r}^{T}
$$

where $\boldsymbol{\Lambda}_{r}$ contains only the 1928 largest eigenvalues, which account for $99.98 \%$ of the cumulative variance of $\widetilde{\boldsymbol{P}}^{b} . \boldsymbol{V}_{r}$ is composed of the corresponding eigenvectors. The reduction of the correlation matrix is equivalent to assuming that the correlations associated with the lowest eigenvalues and corresponding eigenvectors are not meaningful. This technique, described by Cane et al. [1996], not only lightens the computational cost, but also corrects the state of the system only in those directions followed by the dynamical model.

We can now describe the first standard score forecast state as

$$
\begin{aligned}
& \widetilde{\boldsymbol{x}}_{t_{1}}^{f}=\mathbf{0}, \\
& \widetilde{\boldsymbol{P}}_{t_{1}}^{f}=\widetilde{\boldsymbol{P}}_{r}^{b}=\boldsymbol{V}_{r} \boldsymbol{\Lambda}_{r} \boldsymbol{V}_{r}^{T} .
\end{aligned}
$$

\subsection{Analysis}

At every time $t_{i}, i \in\{1, \ldots, n\}$, new observed data $\boldsymbol{y}_{t_{i}}^{o}$ are available. These observations are assumed to be unbiased, which means $\left\langle\boldsymbol{\epsilon}_{t_{i}}^{o}\right\rangle=$ 0. An error covariance matrix $\boldsymbol{R}_{t_{i}}$ is associated with these observations. The observation error covariance matrix $\boldsymbol{R}$ is assumed here to be the same for any time. $\boldsymbol{R}$ and $\boldsymbol{y}_{t_{i}}^{o}$ are standardized with respect to $\boldsymbol{y}^{b}$ and $\boldsymbol{S}_{y}^{b}$

$$
\begin{aligned}
\widetilde{\boldsymbol{y}}_{t_{i}}^{o} & =\left(\boldsymbol{S}_{y}^{b}\right)^{-1}\left(\boldsymbol{y}_{t_{i}}^{o}-\boldsymbol{y}^{b}\right), \\
\widetilde{\boldsymbol{R}} & =\left(\boldsymbol{S}_{y}^{b}\right)^{-1} \boldsymbol{R}\left(\boldsymbol{S}_{y}^{b}\right)^{-1} .
\end{aligned}
$$

Independently, a prior estimate of the state is computed. It is the forecast state $\boldsymbol{x}_{t_{i}}^{f}$, assumed to be unbiased $\left(\left\langle\boldsymbol{\epsilon}_{t_{i}}^{f}\right\rangle=\mathbf{0}\right)$. Its associated error covariance matrix is $\boldsymbol{P}_{t_{i}}^{f} . \quad \boldsymbol{P}_{t_{i}}^{f}$ and $\boldsymbol{x}_{t_{i}}^{f}$ are standardized with respect to $\boldsymbol{x}^{b}$ and $\boldsymbol{S}^{b}$

$$
\begin{aligned}
\widetilde{\boldsymbol{x}}_{t_{i}}^{f} & =\left(\boldsymbol{S}^{b}\right)^{-1}\left(\boldsymbol{x}_{t_{i}}^{f}-\boldsymbol{x}^{b}\right), \\
\widetilde{\boldsymbol{P}}_{t_{i}}^{f} & =\left(\boldsymbol{S}^{b}\right)^{-1} \boldsymbol{P}_{t_{i}}^{f}\left(\boldsymbol{S}^{b}\right)^{-1} .
\end{aligned}
$$

To further simplify this problem, we assume that

$$
\widetilde{\boldsymbol{P}}_{t_{i}}^{f} \approx \widetilde{\boldsymbol{P}}^{b} \approx \boldsymbol{V}_{r} \boldsymbol{\Lambda}_{r} \boldsymbol{V}_{r}^{T} .
$$

Under this assumption, the reduced state Kalman filter is given by

$$
\widetilde{\boldsymbol{K}}_{r}=\left(\widetilde{\boldsymbol{H}} \widetilde{\boldsymbol{P}}_{t_{i}}^{f}\right)^{T}\left[\widetilde{\boldsymbol{H}}\left(\widetilde{\boldsymbol{H}} \widetilde{\boldsymbol{P}}_{t_{i}}^{f}\right)^{T}+\widetilde{\boldsymbol{R}}\right]^{-1} .
$$

The result of the analysis is finally rescaled using the forecast standard deviation

$$
\boldsymbol{x}_{t_{i}}^{a}=\boldsymbol{x}_{t_{i}}^{f}+\boldsymbol{S}_{t_{i}}^{f} \widetilde{\boldsymbol{K}}_{r}\left[\widetilde{\boldsymbol{y}}_{t_{i}}^{o}-\widetilde{\boldsymbol{H}} \widetilde{\boldsymbol{x}}_{t_{i}}^{f}\right] .
$$

\subsection{Forecast}

The forecast state at time $t_{i+1}, \boldsymbol{x}_{t_{i+1}}^{f}$ is computed by the convection code STAGYY, taking $\boldsymbol{x}_{t_{i}}^{a}$ as starting state.

The associated covariance matrix $\boldsymbol{P}_{t_{i+1}}^{f}$ is estimated by assuming

$$
\begin{aligned}
\boldsymbol{P}_{t_{i+1}}^{f} & =\boldsymbol{S}_{t_{i+1}}^{f} \boldsymbol{V}_{r} \boldsymbol{\Lambda}_{r} \boldsymbol{V}_{r}^{T} \boldsymbol{S}_{t_{i+1}}^{f} \\
\boldsymbol{S}_{t_{i+1}}^{f} & =\alpha \boldsymbol{S}_{t_{i}}^{f} .
\end{aligned}
$$

The coefficient $\alpha$ is estimated by considering errors on data and using the fact that the error on forecast data and on observed data are uncorrelated:

$$
\alpha=\sqrt{\frac{\operatorname{Tr}\left(\operatorname{Covar}\left(\boldsymbol{H} \boldsymbol{x}_{t_{i+1}}^{f}-\boldsymbol{y}_{t_{i+1}}^{o}\right)-R\right)}{\operatorname{Tr}\left(\operatorname{Covar}\left(\boldsymbol{H} \boldsymbol{x}_{t_{i}}^{f}-\boldsymbol{y}_{t_{i}}^{o}\right)-R\right)}} .
$$

\section{Synthetic Experiments}

We test here our sequential assimilation algorithm on synthetic cases. The parameters chosen for the model 1 are described in Table 1, along with the parameters of two additional models: model 2, which has a higher bottom heat flux 
to surface heat flux ratio (37\%), and model 3, which has a higher Rayleigh number $\left(10^{7}\right)$. The time in the evolutions computed is rescaled as classically done, using the transit time of the mantle as the relevant scaling time (see Gurnis [1986] for example). The Earth's mantle transit time $t_{t}^{E}$ estimates the average time spent by a particle to move from the surface to the coremantle boundary. For the Earth, it is evaluated by considering the thickness of the mantle $D^{E}$ and the root mean square of surface velocities $v_{r m s}^{E}$

$$
t_{t}^{E}=\frac{D^{E}}{v_{r m s}^{E}} .
$$

$v_{r m s}^{E}$ is obtained from plate tectonics reconstructions (Shephard et al. [2013], Seton et al. [2012], for example). $t_{t}^{M}$, the transit time for the model can be computed by the same procedure. The rescaling of time for a computed evolution is

$$
t=t^{*} \frac{t_{t}^{E}}{t_{t}^{m}},
$$

where $t^{*}$ is the non-dimensional time. This rescaling is designed so that the time of the evolution can compare to that of the Earth, even if the vigour of convection differs.

\subsection{Setup of the Experiments}

We test our data assimilation method on synthetic experiments. They consist of two phases.

First, we synthesize a true state evolution $\left\{\boldsymbol{x}_{1}^{t}, \boldsymbol{x}_{2}^{t}, \ldots, \boldsymbol{x}_{n_{t}}^{t}\right\}$ and corresponding observed data sets $\left\{\boldsymbol{y}_{t_{1}}^{o}, \boldsymbol{y}_{t_{2}}^{o}, \ldots, \boldsymbol{y}_{t_{n}}^{o}\right\}$. This is done by computing a convection evolution spanning $600 \mathrm{My}$ and considering it as the true state evolution $\left\{\boldsymbol{x}_{1}^{t}, \boldsymbol{x}_{2}^{t}, \ldots, \boldsymbol{x}_{n_{t}}^{t}\right\}$. From this evolution, we extract sets of surface heat flow and surface velocities at regular intervals $\Delta t$. These data are randomly noised to produce $\left\{\boldsymbol{y}_{t_{1}}^{o}, \boldsymbol{y}_{t_{2}}^{o}, \ldots, \boldsymbol{y}_{t_{n}}^{o}\right\}$. We set the average amplitude of the random noise as a fraction $\gamma$ of $q_{r m s}$ and $V_{r m s}$, the root mean squares of surface heat flux and velocity, respectively. The observed error covariance matrix is then

$$
\boldsymbol{R}=\gamma^{2}\left(\begin{array}{cccccc}
q_{r m s}^{2} & \ldots & 0 & 0 & \ldots & 0 \\
0 & \ldots & 0 & 0 & \ldots & 0 \\
0 & \ldots & q_{r m s}^{2} & 0 & \ldots & 0 \\
0 & \ldots & 0 & V_{r m s}^{2} & \ldots & 0 \\
0 & \ldots & 0 & 0 & \ldots & V_{r m s}^{2}
\end{array}\right)
$$

Second, we apply the assimilation algorithm using the synthetic data $\left\{\boldsymbol{y}_{t_{1}}^{o}, \boldsymbol{y}_{t_{2}}^{o}, \ldots, \boldsymbol{y}_{t_{n}}^{o}\right\}$. Then, we compare the result of the assimilation, $\left\{\boldsymbol{x}_{1}^{f}, \boldsymbol{x}_{2}^{f}, \ldots, \boldsymbol{x}_{n_{t}}^{f}\right\}$ with the true state $\left\{\boldsymbol{x}_{1}^{t}, \boldsymbol{x}_{2}^{t}, \ldots, \boldsymbol{x}_{n_{t}}^{t}\right\}$.

For each set of model parameters, we compute 21 mantle convection evolutions starting from different initial conditions to produce 21 possible true state evolutions. For model 1 , we test the assimilation for each evolution with 10 different pairs of parameters of data assimilation $(\Delta t, \gamma) . \Delta t$ is varying from $1 \mathrm{My}$ to $50 \mathrm{My}$ and $\gamma$ from $5 \%$ to $50 \%$. For Model 2 and 3, we test the assimilation for each evolution with $\Delta t=10 \mathrm{My}$ and $\gamma=10 \%$. The exact combinations of parameters tested are presented in Table 2. Table 2 defines as well the name of the different assimilations that we will use in the following.

\subsection{Quality of the data assimilation estimate}

We evaluate the quality of the data assimilation scheme on its ability to retrieve the true temperature fields and to match surface data.

Fig. 6 shows examples of the final forecast state (second column) for evolutions with different parameters, after $300 \mathrm{My}$ of data assimilation: $t 10 \gamma 10$ (31 observation times, model 1), $t 50 \gamma 10$ (7 observation times, model 1 ), $t 10 \gamma 10 Q 40$ (31 observation times, model 2) and t10 10 Ra7 (31 observation times, model 3). The two first cases are done using the same model parameters, so we display the data assimilation results of the same evolution for better comparison. The true temperature fields for each case are displayed on the first column. The local error on the third column of Fig. 6 is the absolute value of the error at each coordinate $\left(\phi_{m}, r_{n}\right)$

$$
\epsilon_{T}^{f}\left(\phi_{m}, r_{n}\right)=\left|T^{f}\left(\phi_{m}, r_{n}\right)-T^{t}\left(\phi_{m}, r_{n}\right)\right| .
$$

Below the boundary layer, the locations of positive temperature anomalies are better retrieved for $t 10 \gamma 10$ than for $t 50 \gamma 10$. The general geometry of slabs at the bottom is representative of the true state, even though some details are missing. The geometry of upwellings is retrieved for $t 10 \gamma 10$, but only their approximate location 


\section{Name \\ True State \\ Analyzed State

\author{
Error on \\ Analyzed State
}
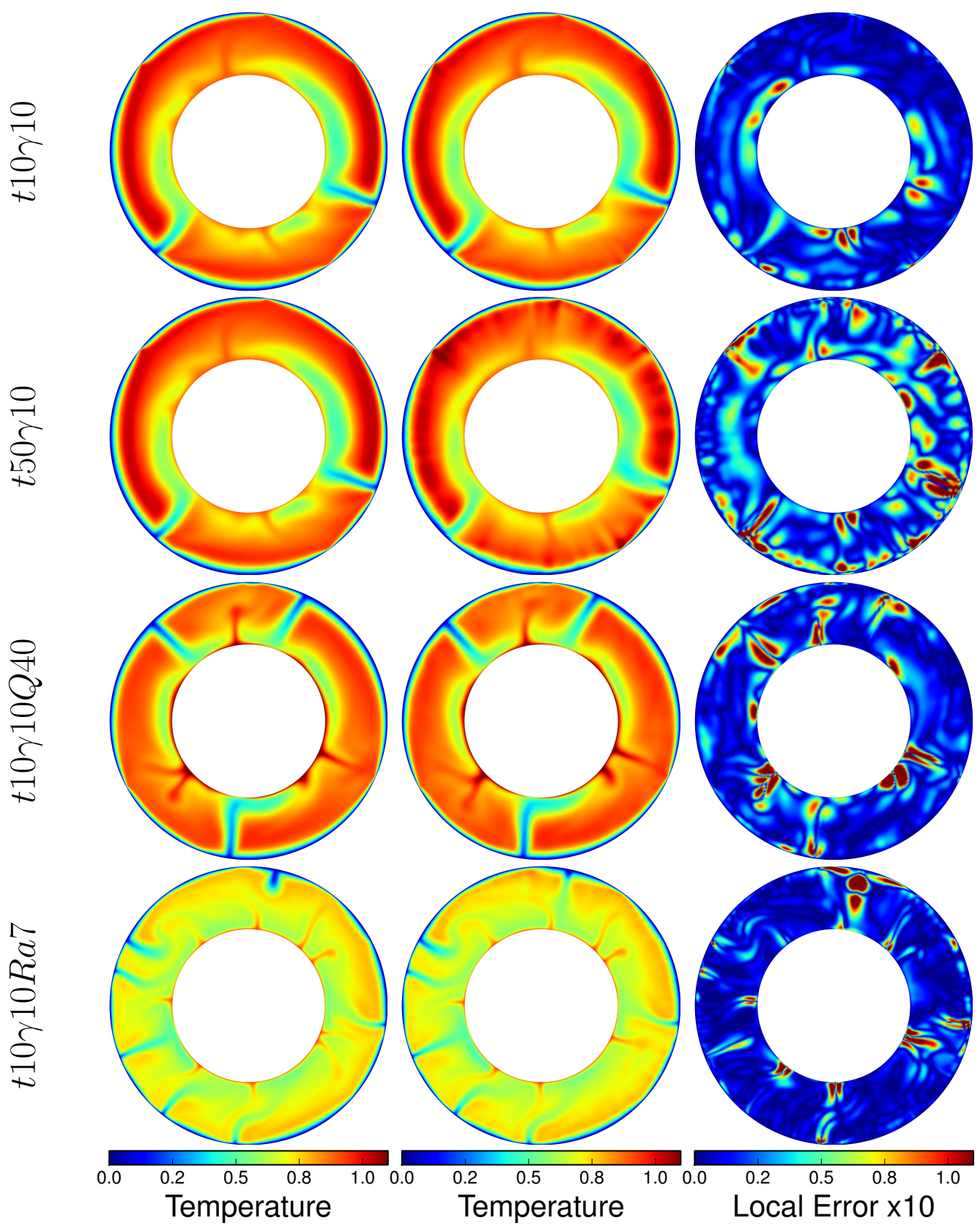

Figure 6: Comparison of the results of data assimilation after $300 \mathrm{My}$ for four cases. First row: data assimilation with a $\Delta t=10 \mathrm{My}$ (experiment $t 10 \gamma 10$ ), second row: data assimilation with a $\Delta t=50 \mathrm{My}$ (experiment $t 50 \gamma 10)$, third row: data assimilation with a $\Delta t=10 \mathrm{My}$ (experiment $t 10 \gamma 10 Q 40$ ) and fourth row: data assimilation with a $\Delta t=10$ My (experiment $t 10 \gamma 10 R a 7$ ). The first column displays the true temperature field for each experiment, the second column shows the analyzed temperature field, and the third column is the associated error multiplied by 10 and represented using the same color scale than the field itself, in order to ease visual inspection. 
Table 2: Values of the parameters of the assimilation for synthetic experiments.

\begin{tabular}{|l|r|r|r|r|r|}
\hline name of experiment & Rayleigh number & Heat flux ratio & $\Delta t(\mathrm{My})$ & $\gamma(\%)$ & number of synthetic experiments \\
\hline$t 1 \gamma 10$ & $10^{6}$ & $10 \%$ & 1 & 10 & 21 \\
\hline$t 5 \gamma 10$ & $10^{6}$ & $10 \%$ & 5 & 10 & 21 \\
\hline$t 10 \gamma 5$ & $10^{6}$ & $10 \%$ & 10 & 5 & 21 \\
\hline$t 10 \gamma 10$ & $10^{6}$ & $10 \%$ & 10 & 10 & 21 \\
\hline$t 10 \gamma 30$ & $10^{6}$ & $10 \%$ & 10 & 30 & 21 \\
\hline$t 10 \gamma 50$ & $10^{6}$ & $10 \%$ & 10 & 50 & 21 \\
\hline$t 15 \gamma 10$ & $10^{6}$ & $10 \%$ & 15 & 10 & 21 \\
\hline$t 20 \gamma 10$ & $10^{6}$ & $10 \%$ & 20 & 10 & 21 \\
\hline$t 50 \gamma 10$ & $10^{6}$ & $10 \%$ & 50 & 10 & 21 \\
\hline$t 10 \gamma 10 Q 40$ & $10^{6}$ & $37 \%$ & 10 & 10 & 21 \\
\hline$t 10 \gamma 10 R a 7$ & $10^{7}$ & $26 \%$ & 10 & 10 & 21 \\
\hline
\end{tabular}

is well estimated for $t 50 \gamma 10$. Some minor positive anomalies are missing at the bottom of the domain for both cases. The relative error shows that although the geometry of the temperature field is reasonably well recovered, the actual temperature values for down- and upwellings are not as accurately estimated. This tendency is especially noticeable for $t 50 \gamma 10$. Overall, the convection pattern is correctly estimated, but for $t 50 \gamma 10$ slabs are blurred. The structures are also well recovered for $t 10 \gamma 10 Q 40$ and $t 10 \gamma 10$ Ra7. We can see that the general shape of the hot upwellings is well estimated for both cases, except on one upwelling, on the bottom right corner of the $t 10 \gamma 10 R a 7$ experiment, where the hot upwelling with two branches is estimated as only one bigger upwelling. The structure of slabs are also reasonnably well estimated. The value of the errors for both $t 10 \gamma 10 Q 40$ and $t 10 \gamma 10 R a 7$ are higher than those of $t 10 \gamma 10$, on some localized regions.

We compute the spatial average error of the temperature field for each timestep $i \in$ $\left\{1,2, \ldots, n_{t}\right\}$

$$
\epsilon_{T}^{f}(i)=\sqrt{\frac{\sum_{m=1}^{M} \sum_{n=1}^{N}\left(T_{i}^{f}\left(\phi_{m}, r_{n}\right)-T_{i}^{t}\left(\phi_{m}, r_{n}\right)\right)^{2} \Delta V\left(\phi_{m}, r_{n}\right)}{\sum_{m=1}^{M} \sum_{n=1}^{N} T_{i}^{t}\left(\phi_{m}, r_{n}\right)^{2} \Delta V\left(\phi_{m}, r_{n}\right)}},
$$

where $\Delta V\left(\phi_{m}, r_{n}\right)$ is the volume of the cell centred on $\left(\phi_{m}, r_{n}\right)$. For each combination of $\Delta t$ and $\gamma, \epsilon_{T}^{f}(i)$ is averaged over 21 synthetic experiments differing only by their initial condi- tions for the model 1 . The synthetic experiments $t 10 \gamma 10 Q 40$ (higher bottom to surface heat flux ratio) and $t 10 \gamma 10 R a 7$ (higher Rayleigh number) showed respectively 20 and 19 successes out of 21. We identify a data assimilation experiment as failing if there is a time after which the global error $\epsilon_{T}^{f}$ is consistently increasing when analyses are performed. The three failure cases show a consistently increasing difference between observed data and forecast data, making them easy to identify even without the knowledge of the true state evolution. Picking another random noise of the same magnitude for each of the three experiments that failed produced a successful data assimilation trial. Hence, peculiar random noise structure could prevent accurate retrieval by the methodology presented here. We compute the average evolution of errors for synthetic experiments $t 10 \gamma 10 Q 40$ and $t 10 \gamma 10 R a 7$ taking into account only the successes, ie 20 experiments for $t 10 \gamma 10 Q 40$ and 19 for $t 10 \gamma 10 R a 7$. Hence, the estimation of the error is not specific to a case that is easier to retrieve than others. On every curve, the analysis times are characterized by a sudden drop of the error (see Figs 7, 8 and 9). This is remarkable for the first analysis, at time 0 , where the error is reduced by a factor of two for any set of parameters tested. These results show that taking into account only the second order statistics is efficient at correcting a forecast state and improving the estimate of the true state. 


\begin{tabular}{|c|c|c|c|c|}
\hline$\longrightarrow$ & $1 \mathrm{My}$ & - & $10 \mathrm{My}$ & - \\
\hline & $5 \mathrm{My}$ & - & $15 \mathrm{My}$ & - \\
\hline
\end{tabular}

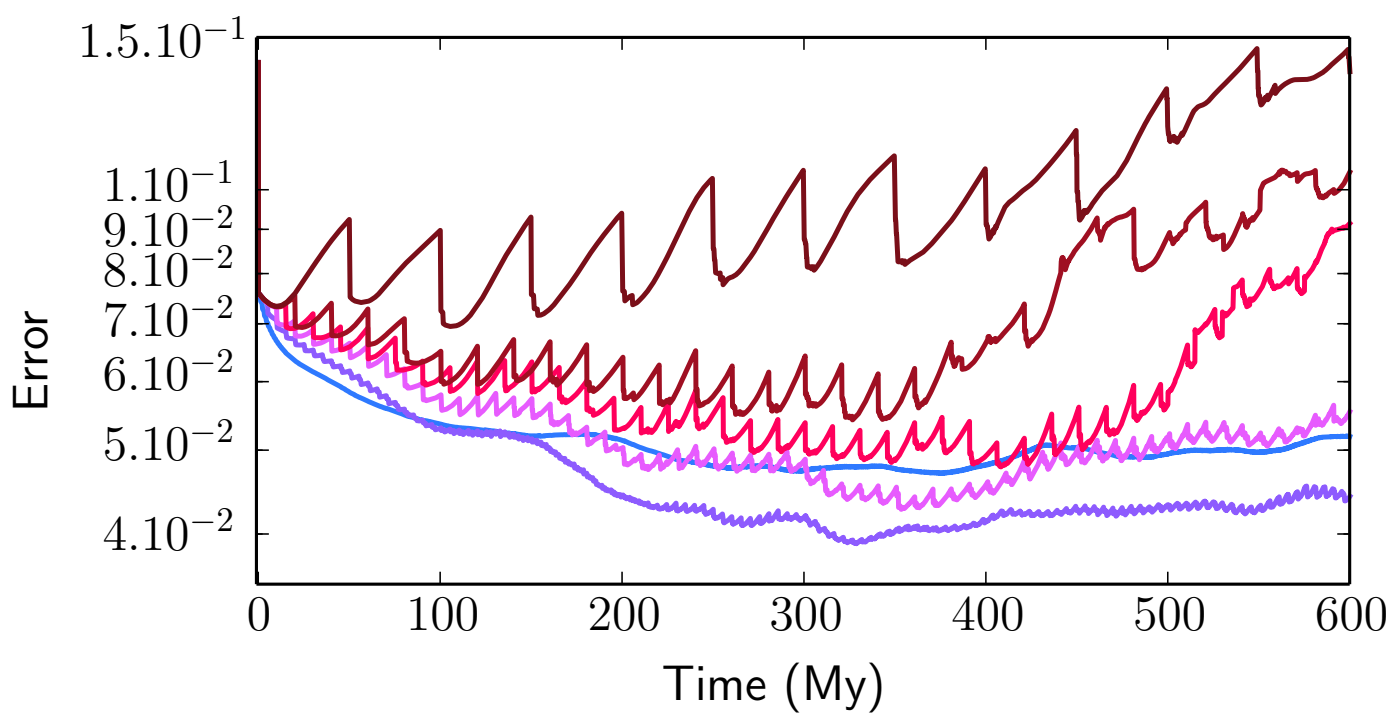

Figure 7: Evolution of the average relative error on the forecast temperature field with $\gamma=10 \%$ and $\Delta t=1,5,10$, 15,20 and $50 \mathrm{My}$. Note that the scale on the y-axis is logarithmic.

As observed in Figs 7,8 and 9, the general evolution of errors with time follows three phases: an error reduction phase, which may be followed by an error stabilization phase around the lower value, and finally an error growth phase. The duration and existence of each phase depends on the chosen combination of $\Delta t, \gamma$ and the model parameters.

Fig. 7 shows the evolutions of errors for data assimilations with noise on observations of $10 \%$ and different frequencies of analyses: $t 1 \gamma 10$, $t 5 \gamma 10, t 10 \gamma 10, t 15 \gamma 10, t 20 \gamma 10$ and $t 50 \gamma 10$. The error reduction phase lasts between 300 to 400 My (2 to 2.5 Lyapunov times) for all cases except for the extreme case of $t 50 \gamma 10$ (50 My representing one third of the Lyapunov time). For the first $100 \mathrm{My}$ of assimilation, the shorter the time between 2 analyses, the faster the errors decrease.

If analysis intervals are equal to or longer than $15 \mathrm{My}$, growth of the error happens within 600 My of assimilation. In contrast, for $t 1 \gamma 10$, $t 5 \gamma 10$ and $t 10 \gamma 10$, no error growth occurs, and errors are stable at around 5\% after $200 \mathrm{My}$. Throughout the assimilation, errors for $t 1 \gamma 10$ become greater than the errors for $t 5 \gamma 10$. This is due to the method used for updating forecast errors in our scheme. Indeed, their estimation is based on the comparison between observed and forecast surface data. A 1 My interval between analyses does not allow the possible errors made internally to be propagated onto the surface. It follows that the error on the forecast is underestimated for short intervals of analysis, leading to results that are not as good as for longer intervals of analysis.

Fig. 8 shows the evolutions of errors for data assimilations with analyses every $10 \mathrm{My}$ and different errors on observations: $t 10 \gamma 5, t 10 \gamma 10$, $t 10 \gamma 30$ and $t 10 \gamma 50$. For levels of noise in observation ranging from 5 to $30 \%$, the error reduction phase lasts more than $300 \mathrm{My}$. Even when observations are noised as much as $50 \%$, data assimilation keeps errors to values lower than $10 \%$ on the estimate of the temperature field over the first $100 \mathrm{My}$. Fig. 8 shows that low levels of noise in observations ( 5 to $10 \%$ ) give results of similar quality.

The error evolutions for $t 10 \gamma 10 R a 7$ and $t 10 \gamma 10 Q 40$ are shown in Fig. 9, along with the 


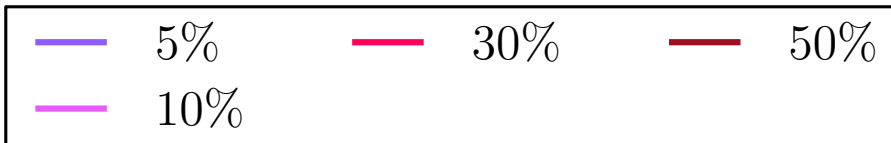

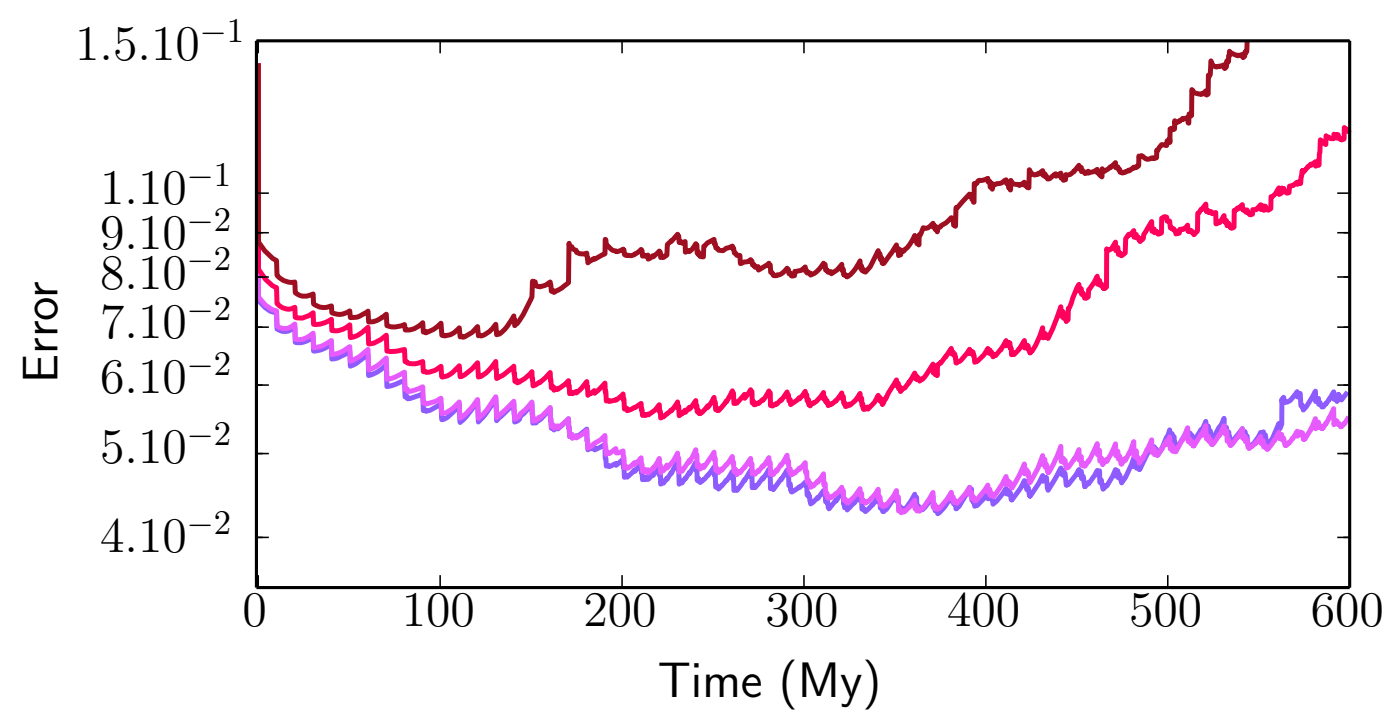

Figure 8: Evolution of the average relative error on the forecast temperature field with $\Delta t=10$ My and $\gamma=5,10$, 30 and $50 \%$. Note that the scale on the $y$-axis is logarithmic.
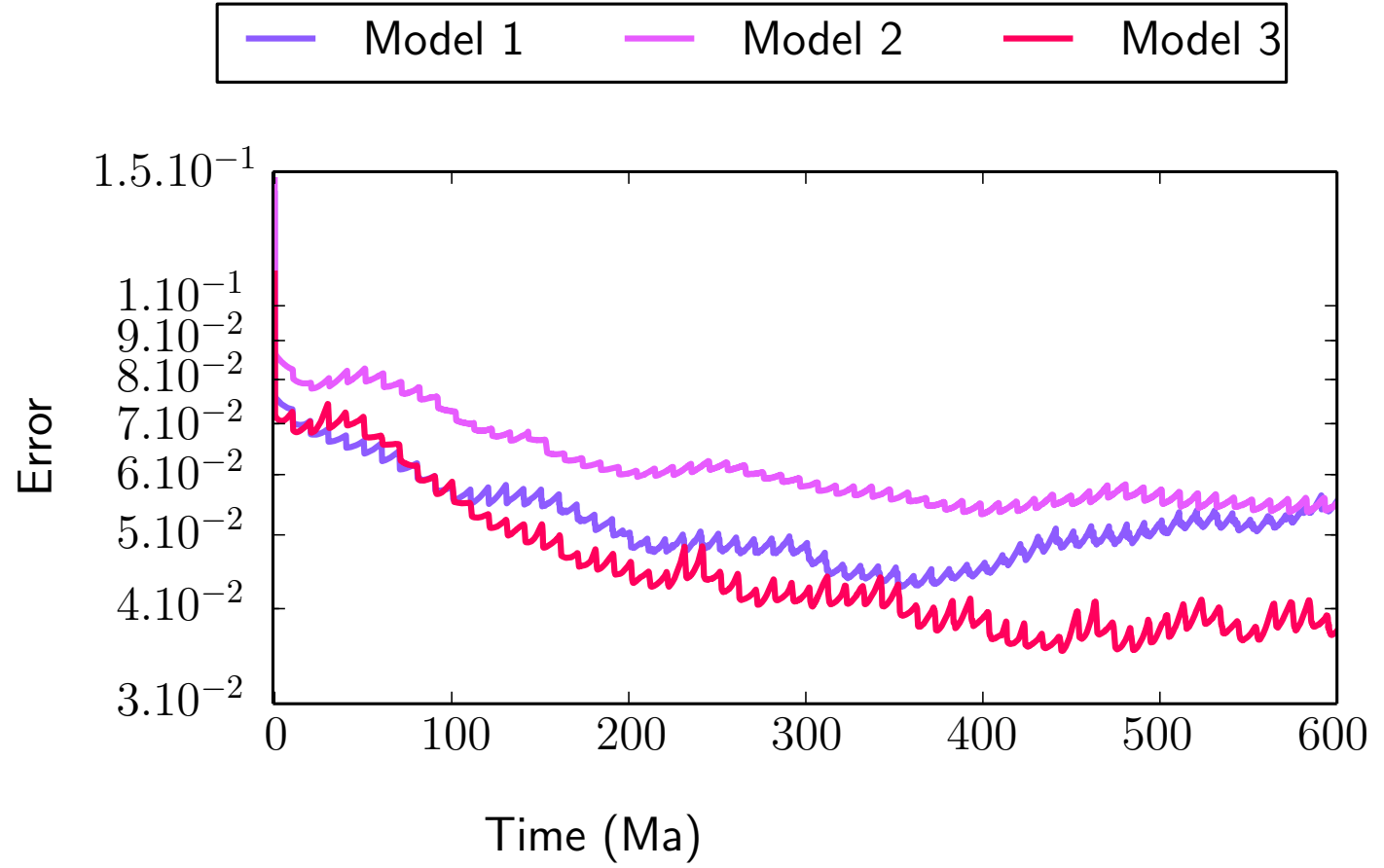

Figure 9: Evolution of the average relative error on the forecast temperature field with $\Delta t=10$ My and $\gamma=10 \%$ for the model 1 , model 2 and model 3 . Note that the scale on the y-axis is logarithmic. 
evolution of errors of $t 10 \gamma 10$. The error for $t 10 \gamma 10 R a 7$ is on average smaller than the error for $t 10 \gamma 10$. On the contrary, the error for $t 10 \gamma 10 Q 40$ is on average bigger than the error of $t 10 \gamma 10$. We note as well that, between two analyses, the errors are increasing much more for $t 10 \gamma 10$ Ra 7 than for the other cases.

Fig. 10 demonstrates that our data assimilation scheme provides a posteriori a better estimate of surface data than the a priori observation for extreme cases. Indeed, for $t 10 \gamma 50$, after $100 \mathrm{My}$ of data assimilation, the a posteriori retrieved surface heat flux and surface velocities are better estimates of the true values than the ones used as the observations.

We conducted here synthetic experiments, where the true state is known, and the result of data assimilation can be compared to this true state evolution. However, in a data assimilation using real observations, the true state of the system is unknown. In this case, it is necessary to use other diagnostics to evaluate the quality of the assimilation. One diagnostic is based on the study of the innovation, which is the difference between the observed data $\boldsymbol{y}_{i}^{o}$ and the forecast data $\boldsymbol{H} \boldsymbol{x}_{i}^{f}$,

$$
\boldsymbol{d}_{i}=\boldsymbol{y}_{i}^{o}-\boldsymbol{H} \boldsymbol{x}_{i}^{f} .
$$

The simplest evaluation is to consider the evolution of the cumulative mean innovation

$$
d_{k}=\left\|\sum_{i=1}^{k} \boldsymbol{d}_{i}\right\|
$$

which is supposed to converge to zero if the scheme is unbiased [Talagrand, 2003].

Fig. 11 shows the cumulative mean innovation for the different parameters, as a function of the number of analyses. The cumulative mean innovation is decreasing through time for all cases, showing that our scheme is unbiased. This should not come as a surprise, since the observations and the model are governed exactly by the same physics.

\section{Discussion}

We chose a sequential method because of its relative ease of implementation compared to alternative methods such as variational data assimilation (see Talagrand [1997], Evensen [2007] for discussions on both methodologies). Moreover, sequential data assimilation has proved to be efficient in solving a wide range of geophysical problems (Aubert and Fournier [2011], Hoteit and Pham [2004] for example). We opted for a suboptimal scheme based on the Kalman filter instead of driving the flow with surface velocities so as to exploit the surface information a step further. The initial condition, or first guess, is given as a compromise between a 1D temperature profile and the inversion of the first data available. Then, the whole temperature field is updated whenever observations are available. This means our models are less sensitive to initial conditions than mantle circulation models in which plate velocities are imposed as boundary conditions.

The specificity of sequential data assimilation is that the estimated state of the mantle improves through time, as more data are assimilated into the model (Fig. 1). This raises an issue since the information at hand for the Earth tends to improve in accuracy as it gets closer to present-day: classical sequential data assimilation schemes are not able to extend the recent information back in time. This difficulty can be overcome by implementing Kalman smoothers [Cohn et al., 1994, Cosme et al., 2010, Nerger et al., 2014] which use time correlations to update the previous states with new observations. Applying these techniques would also enable us to add tomographic models as observations in mantle circulation models, and to propagate this information back in time. However, one of the benefits of the method we developped would be to provide an image of the present state of the Earth mantle which is independant from seismic data, and would give the opportunity to work on the interpretation of tomographic models.

Our sequential assimilation technique gives encouraging results for our test cases: data assimilation gradually reduces the distance between the true models and the estimated ones for the first $300 \mathrm{My}$, even for highly noised data (up to $30 \%$ of noise). The analysis is based on a linear correction, the amplitude of which depends on the distance between the predicted data and the observed data. The linearity of the correction is an approximation that is efficient as soon as the prediction is close enough to the ob- 


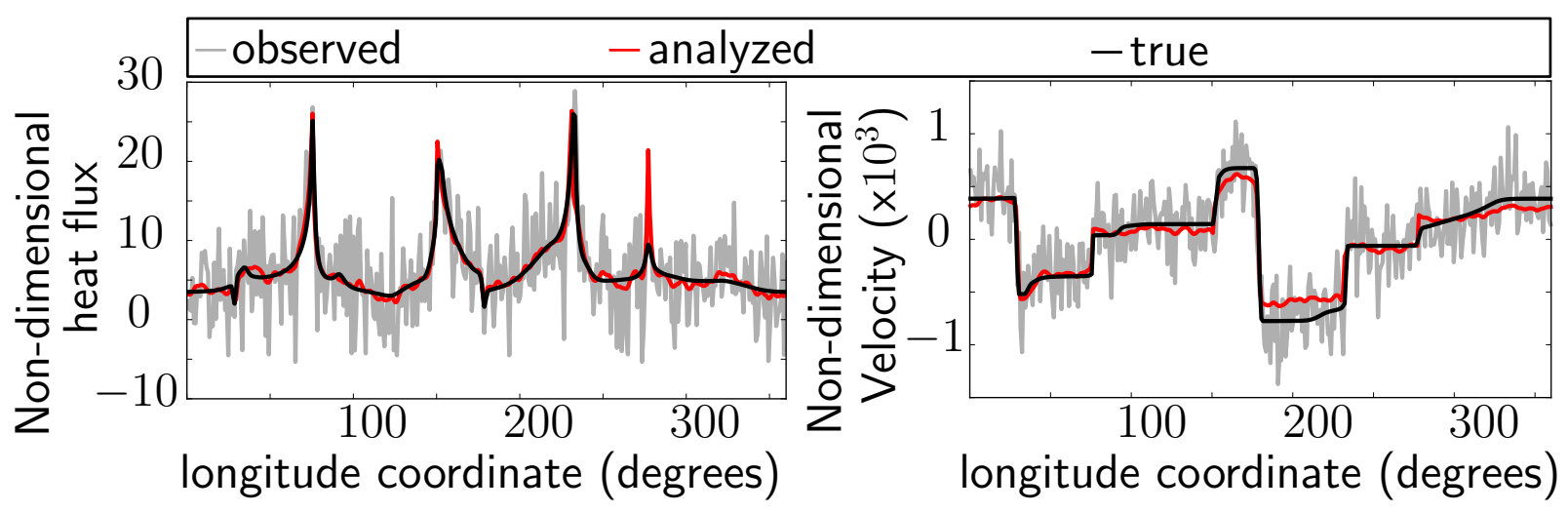

Figure 10: Comparison between true, observed and analyzed surface heat flux (left) and surface velocities (right) after $100 \mathrm{My}$ of assimilation with the parameters $t 10 \gamma 50$
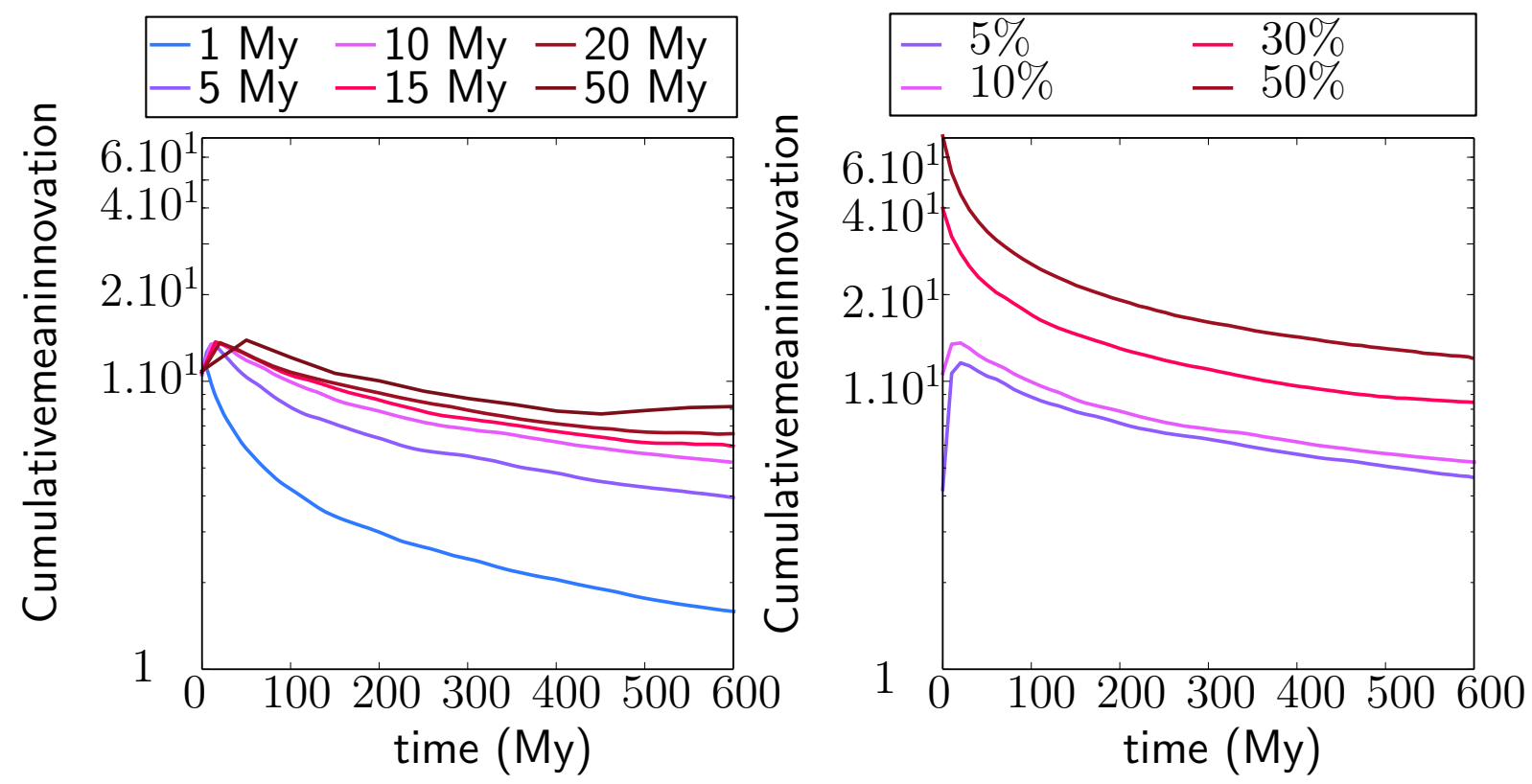

Figure 11: Evolution of the cumulative mean innovation as a function of the number of analyses. On the left: for model 1 with $\gamma=10 \%$ and $\Delta t=1,5,10,15,20$ and $50 \mathrm{My}$; on the right: for Model 1 with $\Delta t=10 \mathrm{My}$ and $\gamma=5$, 10,30 and $50 \%$ 
servation. When these two are too different, the analysis tends to over-correct the state, which leads to an unrealistic analyzed state. The algorithm is stable as long as the time between two observations is short enough: if it is $15 \mathrm{My}$ and greater the estimated temperature field will eventually diverge from the true temperature field. If the time between two analyses is too long, the linear approximation for correcting the state field is not valid any more. The temperature field is over-corrected and this leads to analyzed temperatures inconsistent with the physical model.

We evaluate hereafter how the required $\Delta t$ and $\gamma$ in our test cases compare to the corresponding values available for Earth's data: time between two plate reconstruction stages and uncertainty on plate kinematics and seafloor age maps. The seafloor spreading isochrons used for tectonic reconstructions are based on the identification of magnetic anomalies Müller et al., 2008. This gives a strong constraint on the motion of plates at least every $10 \mathrm{My}$, except for the Cretaceous superchron spanning between 83.5 to $125 \mathrm{Ma}$. Other geological arguments have been provided to infer plate evolutions for this period [Torsvik et al., 2009]. For more recent times (the last $20 \mathrm{My}$ ), more precise surveys of magnetic anomalies have led to regional reconstructions with a temporal resolution of around 1 My Merkouriev and DeMets, 2006]. Moreover, Gurnis et al. [2012] developed a way to estimate kinematic states between two stages of reconstructions. With this method, having data to assimilate at least every $10 \mathrm{My}$ is ensured, although interpolated solutions could miss peculiar changes in plate motions, and errors on the reconstructed states propagate into the interpolated states.

Regarding these errors, noise in plate tectonic reconstructions is difficult to estimate since the process involves taking into account various types of data and human syntheses. Hence, uncertainties on plate reconstructions increase as we go back in time, since less data are available and more interpretation is required. The accuracy of reconstructions also varies in space. For instance the evolution of the Atlantic for more than $100 \mathrm{My}$ is well known, however, the eastern Pacific before $60 \mathrm{My}$ is mostly unknown because the oceanic seafloor has subducted. Efforts to estimate errors on plate tectonic reconstructions have been directed to the estimation of errors on present maps of oceanic sea floor Müller et al. 2008], and on the noise reduction in finite rotations deduced from them laffaldano et al. 2014]. Both of these works show that the error of age maps is smaller than $10 \%$ for recent times $(<50 \mathrm{My})$. However, errors back in time are more difficult to estimate since accuracy for certain plates decreases (like those in the eastern Pacific as discussed before for instance), and an error on the angular velocity or the position of the Euler pole would constrain the errors on velocities over the whole plate domain Molnar and Stock, 1985]. These errors would have to be propagated to seafloor age maps.

As successful as it may appear, the method presented here has several limitations. First, our technique does not take into account nonlinearities of the model physics, and the nonGaussianity of the distribution of temperature, heat flux and velocity values. Indeed, we computed only second order statistics to estimate the link between temperatures and velocities. However, the method we present here already captures the essential features of mantle convection, and provides accurate convection reconstructions with the approximations made.

In this study, we assumed a perfect model (with $\eta=0$ as stated in Equation 12). However, this would not be the case for applying the technique with Earth data. Model equations assume simplifications of the physics, detailed in Ricard [2007]. Any parametrization we may use would have shortcomings. The most important, least known, ingredient is thought to be rheology, which is a strong limitation according to Worthen et al. [2014]. Applying our method to Earth data would help evaluate the forecasting power of the state-of-the-art convection models. Explicitly introducing a model error term in the data assimilation scheme could be a way to improve the reconstructions [Evensen, 2007]. Also, we used heat flux as a proxy for seafloor age because there is no small scale convection in our test cases. For Earth data, monitoring age with tracers, giving the exact estimate of seafloor age would be required. The state vector and observation matrix would differ slightly, 
but the technique would still apply. Complexifying the direct model used in data assimilation (ie having a 3D model at higher Rayleigh number, with a more complex rheology and a multi component system) will as well increase dramatically the size of the data assimilation problem. In particular, the state covariance matrix will reach a size at which the direct computations carried out in this paper will be extremely heavy. One solution would be to take advantage of the structure and properties of the covariance matrix (symmetries, periodicity) to design faster algorithms. The method could also be modified to avoid the computation of the covariance matrix and calculate directly the smaller matrix $\widetilde{\boldsymbol{H}} \widetilde{\boldsymbol{P}}_{t_{i}}^{f}$.

\section{Conclusion}

We have applied sequential data assimilation methodology to reconstruct mantle convection using surface data (velocity and heat flux as a proxy for seafloor age). This work is a first approach to test the efficiency of assimilating surface data to recover the evolution of convecting structures. Our technique belongs to the suboptimal schemes of sequential data assimilation based on the Kalman filter. We modified the optimal interpolation method to update the forecast error covariance matrix.

Our scheme of sequential data assimilation proves to be efficient in recovering the temperature field of a convective system with plate-like tectonics at its surface over several $100 \mathrm{My}$. The only observations used were surface heat fluxes and surface velocities. We tested the robustness of the method by conducting synthetic experiments in 2D spherical annulus geometry. We obtained accurate results, even for the location of plumes at the base of the models, for periods of at least 300 My provided the time between analyses is shorter than $15 \mathrm{My}$ and the noise in observation is lower than $30 \%$. These requirements are satisfactory, since current plate reconstruction models already provide estimates for velocities and ages at least every $10 \mathrm{My}$ and with a fine accuracy for the past $60 \mathrm{My}$, and possibly beyond.

Application to the Earth would first involve the use of 3D-spherical sophisticated models, with high resolution, high convective vigour, continents and a more realistic rheology. In 3D the ratio of number of data points to unknowns would be the same as in 2D spherical annulus geometry by definition. However, increasing the resolution of our model would make this ratio deteriorate, which could be compensated by increasing the correlation between closer nodes. Moreover, although convection models can account for complex parametrizations, their limitations could introduce errors in the reconstructions.

These difficulties should not stop the exploitation of the method: if successful, the convection reconstruction would provide a new image of the mantle, alternative to seismic tomography, which would appear as an independent source of information and assess the quality of the estimate of the state of the mantle at present day; if unsuccessful, it would provide a quantitative evaluation of forward models and help us decide how to improve them.

\section{ACKNOWLEDGMENTS}

We like to thank the editor as well as the two anonymous reviewers for their comments that helped to improve the initial manuscript. The research leading to these results has received funding from the European Research Council within the framework of the SP2-Ideas Program ERC2013-CoG, under ERC grant agreement 617588. Calculations were performed on LGLTPE Seisglob high-performance computing cluster and using HPC resources from GENCI-IDRIS (grant 2014-047243). Figures were generated using matplotlib library Hunter [2007]. The contribution of Alexandre Fournier is IPGP contribution number 3675 . 


\section{References}

Alisic, L., Gurnis, M., Stadler, G., Burstedde, C., Ghattas, O., 2012. Multi-scale dynamics and rheology of mantle flow with plates. Journal of Geophysical Research: Solid Earth (1978-2012) 117 (B10).

Aubert, J., Fournier, A., 2011. Inferring internal properties of earths core dynamics and their evolution from surface observations and a numerical geodynamo model. Nonlin. Processes Geophys 18, 657-674.

Balachandar, S., 1998. Eigendecomposition of the twopoint correlation tensor for optimal characterization of mantle convection. Geophysical journal international 132 (1), 111-127.

Bello, L., Coltice, N., Rolf, T., Tackley, P. J., 2014. On the predictability limit of convection models of the earth's mantle. Geochemistry, Geophysics, Geosystems.

Bercovici, D., 2003. The generation of plate tectonics from mantle convection. Earth and Planetary Science Letters 205 (3), 107-121.

Bercovici, D., Ricard, Y., 2014. Plate tectonics, damage and inheritance. Nature 508 (7497), 513-516.

Bower, D. J., Gurnis, M., Flament, N., 2015. Assimilating lithosphere and slab history in 4-d earth models. Physics of the Earth and Planetary Interiors 238, 8-22.

Bunge, H.-P., Grand, S. P., 2000. Mesozoic plate-motion history below the northeast pacific ocean from seismic images of the subducted farallon slab. Nature 405 (6784), 337-340.

Bunge, H.-P., Hagelberg, C., Travis, B., 2003. Mantle circulation models with variational data assimilation: inferring past mantle flow and structure from plate motion histories and seismic tomography. Geophysical Journal International 152 (2), 280-301.

Bunge, H.-P., Richards, M., Baumgardner, J., 2002. Mantle-circulation models with sequential data assimilation: inferring present-day mantle structure from plate-motion histories. Philosophical Transactions of the Royal Society of London. Series A: Mathematical, Physical and Engineering Sciences 360 (1800), 25452567.

Bunge, H.-P., Richards, M. A., Lithgow-Bertelloni, C., Baumgardner, J. R., Grand, S. P., Romanowicz, B. A., 1998. Time scales and heterogeneous structure in geodynamic earth models. Science 280 (5360), 91-95.

Cane, M. A., Kaplan, A., Miller, R. N., Tang, B., Hackert, E. C., Busalacchi, A. J., 1996. Mapping tropical pacific sea level: Data assimilation via a reduced state space kalman filter. Journal of Geophysical Research: Oceans (1978-2012) 101 (C10), 22599-22617.

Cohn, S. E., Sivakumaran, N., Todling, R., 1994. A fixedlag kalman smoother for retrospective data assimilation. Monthly Weather Review 122 (12), 2838-2867.

Coltice, N., Rolf, T., Tackley, P., Labrosse, S., 2012. Dynamic causes of the relation between area and age of the ocean floor. Science 336 (6079), 335-338.

Coltice, N., Seton, M., Rolf, T., Müller, R., Tackley, P. J., 2013. Convergence of tectonic reconstructions and mantle convection models for significant fluctuations in seafloor spreading. Earth and Planetary Science Letters 383, 92-100.
Cosme, E., Brankart, J.-M., Verron, J., Brasseur, P., Krysta, M., 2010. Implementation of a reduced rank square-root smoother for high resolution ocean data assimilation. Ocean Modelling 33 (1), 87-100.

Evensen, G., 2007. Data assimilation. Springer.

Flament, N., Gurnis, M., Müller, R. D., 2013. A review of observations and models of dynamic topography. Lithosphere 5 (2), 189-210.

Fournier, A., Hulot, G., Jault, D., Kuang, W., Tangborn, A., Gillet, N., Canet, E., Aubert, J., Lhuillier, F., 2010. An introduction to data assimilation and predictability in geomagnetism. Space science reviews 155 (1-4), 247-291.

Ghil, M., Malanotte-Rizzoli, P., 1991. Data assimilation in meteorology and oceanography. Advances in geophysics 33, 141-266.

Gurnis, M., 1986. Stirring and mixing in the mantle by plate-scale flow: Large persistent blobs and long tendrils coexist. Geophysical Research Letters 13 (13), 1474-1477.

Gurnis, M., Turner, M., Zahirovic, S., DiCaprio, L., Spasojevic, S., Müller, R. D., Boyden, J., Seton, M., Manea, V. C., Bower, D. J., 2012. Plate tectonic reconstructions with continuously closing plates. Computers \& Geosciences 38 (1), 35-42.

Hernlund, J. W., Tackley, P. J., 2008. Modeling mantle convection in the spherical annulus. Physics of the Earth and Planetary Interiors 171 (1), 48-54.

Hoteit, I., Pham, D.-T., 2004. An adaptively reducedorder extended kalman filter for data assimilation in the tropical pacific. Journal of marine systems 45 (3), 173-188.

Hunter, J. D., 2007. Matplotlib: A 2d graphics environment. Computing In Science \& Engineering 9 (3), 90-95.

laffaldano, G., Hawkins, R., Bodin, T., Sambridge, M., 2014. Redback: Open-source software for efficient noise-reduction in plate kinematic reconstructions. Geochemistry, Geophysics, Geosystems 15 (4), 1663-1670.

Ide, K., Courtier, P., Ghil, M., Lorenc, A., 1997. Unified notation for data assimilation: operational, sequential and variational. Practice 75 (1B), 181-189.

Ismail-Zadeh, A., Korotkii, A., Schubert, G., Tsepelev, I., 2007. Quasi-reversibility method for data assimilation in models of mantle dynamics. Geophysical Journal International 170 (3), 1381-1398.

Ismail-Zadeh, A., Tackley, P., 2010. Computational methods for geodynamics. Cambridge University Press.

Kalnay, E., 2003. Atmospheric modeling, data assimilation, and predictability. Cambridge university press.

Liu, L., Gurnis, M., 2008. Simultaneous inversion of mantle properties and initial conditions using an adjoint of mantle convection. Journal of Geophysical Research: Solid Earth (1978-2012) 113 (B8).

Merkouriev, S., DeMets, C., 2006. Constraints on indian plate motion since 20 ma from dense russian magnetic data: Implications for indian plate dynamics. Geochemistry, Geophysics, Geosystems 7 (2).

Molnar, P., Stock, J. M., 1985. A method for bounding uncertainties in combined plate reconstructions. Jour- 
nal of Geophysical Research: Solid Earth (1978-2012) 90 (B14), 12537-12544.

Moresi, L., Gurnis, M., Zhong, S., 2000. Plate tectonics and convection in the earths mantle: Toward a numerical simulation. Computing in Science \& Engineering 2 (3), 22-33.

Moresi, L., Solomatov, V., 1998. Mantle convection with a brittle lithosphere: thoughts on the global tectonic styles of the earth and venus. Geophysical Journal International 133 (3), 669-682.

Müller, R. D., Sdrolias, M., Gaina, C., Roest, W. R., 2008. Age, spreading rates, and spreading asymmetry of the world's ocean crust. Geochemistry, Geophysics, Geosystems 9 (4)

Nerger, L., Schulte, S., Bunse-Gerstner, A., 2014. On the influence of model nonlinearity and localization on ensemble kalman smoothing. Quarterly Journal of the Royal Meteorological Society 140 (684), 2249-2259.

Ricard, Y., 2007. Physics of mantle convection. Treatise on Geophysics 7, 31-87.

Ricard, Y., Vigny, C., Froidevaux, C., 1989. Mantle heterogeneities, geoid, and plate motion: A monte carlo inversion. Journal of Geophysical Research: Solid Earth (1978-2012) 94 (B10), 13739-13754.

Richards, M. A., Yang, W.-S., Baumgardner, J. R., Bunge, H.-P., 2001. Role of a low-viscosity zone in stabilizing plate tectonics: Implications for comparative terrestrial planetology. Geochemistry, Geophysics, Geosystems 2 (8)

Rolf, T., Coltice, N., Tackley, P., 2014. Statistical cyclicity of the supercontinent cycle. Geophysical Research Letters 41 (7), 2351-2358.

Seton, M., Müller, R., Zahirovic, S., Gaina, C., Torsvik, T., Shephard, G., Talsma, A., Gurnis, M., Turner, M., Maus, S., et al., 2012. Global continental and ocean basin reconstructions since $200 \mathrm{ma}$. Earth-Science Reviews 113 (3), 212-270.

Shephard, G. E., Müller, R. D., Seton, M., 2013. The tectonic evolution of the arctic since pangea breakup: Integrating constraints from surface geology and geophysics with mantle structure. Earth-Science Reviews 124, 148-183.

Solomatov, V., 1995. Scaling of temperature-and stressdependent viscosity convection. Physics of Fluids (1994-present) 7 (2), 266-274.

Stein, C., Schmalzl, J., Hansen, U., 2004. The effect of rheological parameters on plate behaviour in a selfconsistent model of mantle convection. Physics of the Earth and Planetary Interiors 142 (3), 225-255.

Tackley, P. J., 2000. Self-consistent generation of tectonic plates in time-dependent, three-dimensional mantle convection simulations. Geochemistry, Geophysics, Geosystems 1 (8).

Tackley, P. J., Stevenson, D. J., Glatzmaier, G. A., Schubert, G., 1993. Effects of an endothermic phase transition at $670 \mathrm{~km}$ depth in a spherical model of convection in the earth's mantle. Nature 361 (6414), 699704.

Talagrand, O., 1997. Assimilation of observations, an introduction. Journal-Meteorological Society of Japan Series 2 75, 81-99.

Talagrand, O., 2003. A posteriori validation of assimi- lation algorithms. In: Data assimilation for the earth system. Springer, pp. 85-95.

Todling, R., Cohn, S. E., 1994. Suboptimal schemes for atmospheric data assimilation based on the kalman filter. Monthly Weather Review 122 (11), 2530-2557.

Torsvik, T. H., Rousse, S., Labails, C., Smethurst, M. A., 2009. A new scheme for the opening of the south atlantic ocean and the dissection of an aptian salt basin. Geophysical Journal International 177 (3), 1315-1333.

Van Heck, H., Tackley, P., 2008. Planforms of selfconsistently generated plates in $3 \mathrm{~d}$ spherical geometry. Geophysical Research Letters 35 (19).

Worthen, J., Stadler, G., Petra, N., Gurnis, M., Ghattas, O., 2014. Towards adjoint-based inversion for rheological parameters in nonlinear viscous mantle flow. Physics of the Earth and Planetary Interiors 234, 2334.

Wunsch, C., 2006. Discrete inverse and state estimation problems: with geophysical fluid applications. Vol. 2. Cambridge University Press Cambridge.

Zhong, S., 2001. Role of ocean-continent contrast and continental keels on plate motion, net rotation of lithosphere, and the geoid. Journal of Geophysical Research: Solid Earth (1978-2012) 106 (B1), 703-712. 